\title{
Photodynamic Therapy Combined with Antibiotics or Antifungals against Microorganisms That Cause Skin and Soft Tissue Infections: A Planktonic and Biofilm Approach to Overcome Resistances
}

\author{
Vanesa Pérez-Laguna ${ }^{1, *(D)}$, Isabel García-Luque ${ }^{2}$ (D) , Sofía Ballesta ${ }^{2}$, Antonio Rezusta ${ }^{3,4,5,+}$ \\ and Yolanda Gilaberte ${ }^{3,6,+}$ \\ 1 Max Planck Institute for Evolutionary Biology, 24306 Plön, Germany \\ 2 Department of Microbiology, School of Medicine, University of Sevilla and Instituto de Biomedicina de \\ Sevilla (IBIS), 41009 Sevilla, Spain; igarcial@us.es (I.G.-L.); mudarra@us.es (S.B.) \\ 3 Aragon Health Research Institute (IIS Aragón), 50009 Zaragoza, Spain; arezusta@unizar.es (A.R.); \\ ygilaberte@salud.aragon.es (Y.G.) \\ 4 Department of Microbiology, Miguel Servet University Hospital, 50009 Zaragoza, Spain \\ 5 Department of Microbiology, Preventive Medicine and Public Health, University of Zaragoza, \\ 50001 Zaragoza, Spain \\ 6 Department of Dermatology, Miguel Servet University Hospital, 50009 Zaragoza, Spain \\ * Correspondence: perezlaguna@evolbio.mpg.de \\ $+\quad$ These authors contributed equally to this work.
}

Citation: Pérez-Laguna, V.;

García-Luque, I.; Ballesta, S.; Rezusta,

A.; Gilaberte, Y. Photodynamic

Therapy Combined with Antibiotics

or Antifungals against

Microorganisms That Cause Skin and

Soft Tissue Infections: A Planktonic and Biofilm Approach to Overcome Resistances. Pharmaceuticals 2021, 14, 603. https://doi.org/10.3390/ ph14070603

Academic Editor: Serge Mordon

Received: 15 May 2021

Accepted: 16 June 2021

Published: 23 June 2021

Publisher's Note: MDPI stays neutral with regard to jurisdictional claims in published maps and institutional affiliations.

Copyright: (c) 2021 by the authors. Licensee MDPI, Basel, Switzerland. This article is an open access article distributed under the terms and conditions of the Creative Commons Attribution (CC BY) license (https:// creativecommons.org/licenses/by/ $4.0 /)$.

\begin{abstract}
The present review covers combination approaches of antimicrobial photodynamic therapy (aPDT) plus antibiotics or antifungals to attack bacteria and fungi in vitro (both planktonic and biofilm forms) focused on those microorganisms that cause infections in skin and soft tissues. The combination can prevent failure in the fight against these microorganisms: antimicrobial drugs can increase the susceptibility of microorganisms to aPDT and prevent the possibility of regrowth of those that were not inactivated during the irradiation; meanwhile, aPDT is effective regardless of the resistance pattern of the strain and their use does not contribute to the selection of antimicrobial resistance. Additive or synergistic antimicrobial effects in vitro are evaluated and the best combinations are presented. The use of combined treatment of aPDT with antimicrobials could help overcome the difficulty of fighting high level of resistance microorganisms and, as it is a multi-target approach, it could make the selection of resistant microorganisms more difficult.
\end{abstract}

Keywords: photoinactivation; resistance; antimicrobial agents; SSTI; MDR

\section{Introduction}

\subsection{The Problem of Skin and Soft Tissue Infections}

Skin and soft tissue infections (SSTIs) are defined as clinical entities of variable presentation, etiology, and severity that involve microbial invasion of the layers of the skin and underlying soft tissues. Related to clinical manifestations of the wide range of pathologies they represent, the minimum diagnostic criteria are erythema, edema, warmth, and pain or tenderness. The affected area may also become dysfunctional depending on the severity of the infection, and, much more relevantly, patient comorbidity can easily transform a normally mild infection into a rapidly advancing threat to life [1-3]. Complicated forms of SSTI (cSSTI) may need, apart from antibiotic or antifungal treatment, surgical procedures, or have significant underlying co-morbidities such as diabetes, systemic immunosuppression, or neurological diseases [4-6].

Their treatment has become more challenging because of the increase in the frequency and severity of infections mainly due to the ageing of the general population, the increased number of critical and immunocompromised patients, and because of the emergence 
of resistance to many of the antimicrobial agents commonly used to treat SSTIs in the past [7-9].

\subsection{Antimicrobial Resistance in Skin and Soft Tissue Infections Causal Agents}

Antimicrobial resistance (AMR) makes treatments more tedious and it has adverse consequences such as prolonged hospitalization, increased medical expenses, overburdened public health system, and even increased mortality rates [10].

The main causative agent of SSTI is Staphylococcus aureus, which is one of the bacteria most frequently involved in the problem of AMR. Other causal agents of a high percentage of SSTI, also involved in AMR problem, are Enterobacteriaceae and Pseudomonas aeruginosa (e.g., $45.9 \%$ of hospital-acquired SSTI in North America are caused by S. aureus, $10.8 \%$ by P. aeruginosa, and $8.2 \%$ by Enterococcus spp.) [1]. In fact, in 2017 the World Health Organization (WHO) published the first list of bacteria for which new antibiotics were urgently needed [11] (Table 1). Carbapenem-resistant or third generation cephalosporin-resistant Enterobacteriaceae (e.g., Escherichia coli) and carbapenem-resistant P. aeruginosa are classified in this list within the most dangerous class (Priority 1: critical) and methicillin-resistant or vancomycin intermediate and resistant S. aureus is classified as "Priority 2: high".

Table 1. Global priority list of antibiotic-resistant bacteria (Adapted from: https:/ /www.who.int/ medicines / publications/WHO-PPL-Short_Summary_25Feb-ET_NM_WHO.pdf?ua=1 (accessed on 20 June 2021)).

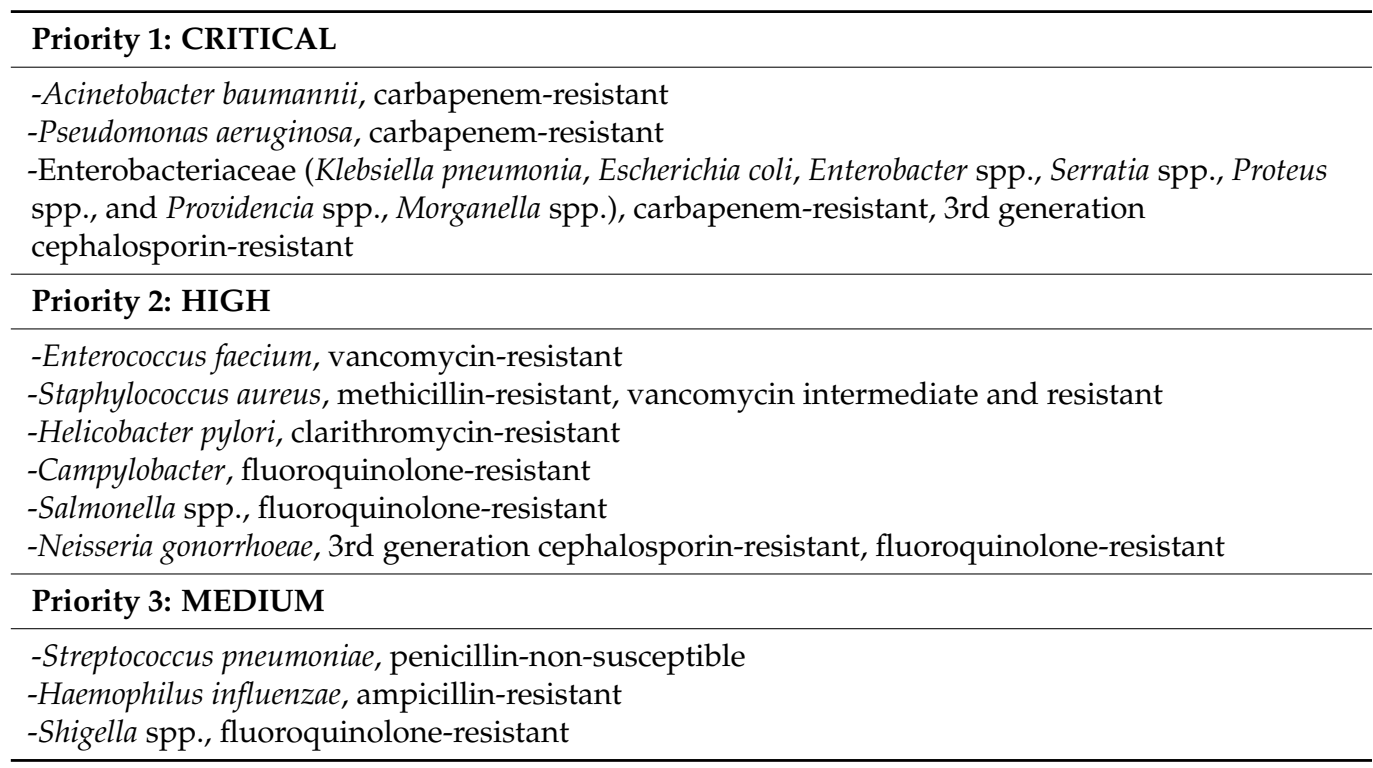

Carbapenem-resistant Acinetobacter baumannii completes the list of bacteria classified as "Priority 1". A. baumannii-associated SSTI is an emerging infection in patients who experience trauma; although it causes few cases, usually they are severe [12].

Regarding atypical mycobacteria, which also cause SSTI on certain occasions, they are highly resistant to antibacterial treatments due to the special characteristics of their wall (the mycobacterial cell has four main layers: (i) the plasma membrane, (ii) the peptidoglycanarabinogalactan complex, (iii) an asymmetrical outer membrane covalently linked with mycolic acids, and (iv) an outermost capsule). They are a globally established priority for which innovative new treatments are urgently needed according to the WHO [11].

On the other hand, the recent emergence of fungi that are resistant to more than one class of antifungal drug is a serious concern, especially because currently only three primary classes of agents are used to treat invasive fungal infections: (1) Azoles (Fluconazole, voriconazole, posaconazole), (2) Echinocandins (Caspofungin, micafungin, anidulafungin) and (3) Polyne (Amphotericin B). Candida glabrata, Candida krusei and Candida auris are species with intrinsic or high rates of resistance against the first, Cryptococcus spp. and 
Fusarium spp. against the second, and Candida auris and Aspergillus terreus against the last [13].

With regard to yeasts that cause SSTI, Candida spp. stand out as causative agents. They are implicated in the AMR problem due to the increasingly frequent existence of fluconazole resistant strains [13,14].

Finally, dermatophytes and non-dermatophytes molds cause cutaneous and subcutaneous infections than are often chronic and recalcitrant. Invasive infections are rare but occur especially in immunocompromised and debilitated individuals. Its treatment is a great challenge especially in these patients. $[15,16]$.

\subsection{Antimicrobial Photodynamic Therapy Combined with Antibiotics or Antifungals to Treat SSTIs}

Antimicrobial photodynamic therapy (aPDT) is based on the use of photosensitizer molecules (PS) that are activated by light in the presence of oxygen. Reactive oxygen species (ROS) are generated in the photodynamic reaction resulting in a toxic effect for microorganisms that is capable of destroying them (Figure 1). Hence, aPDT has been proposed as an alternative treatment for SSTIs $[17,18]$.

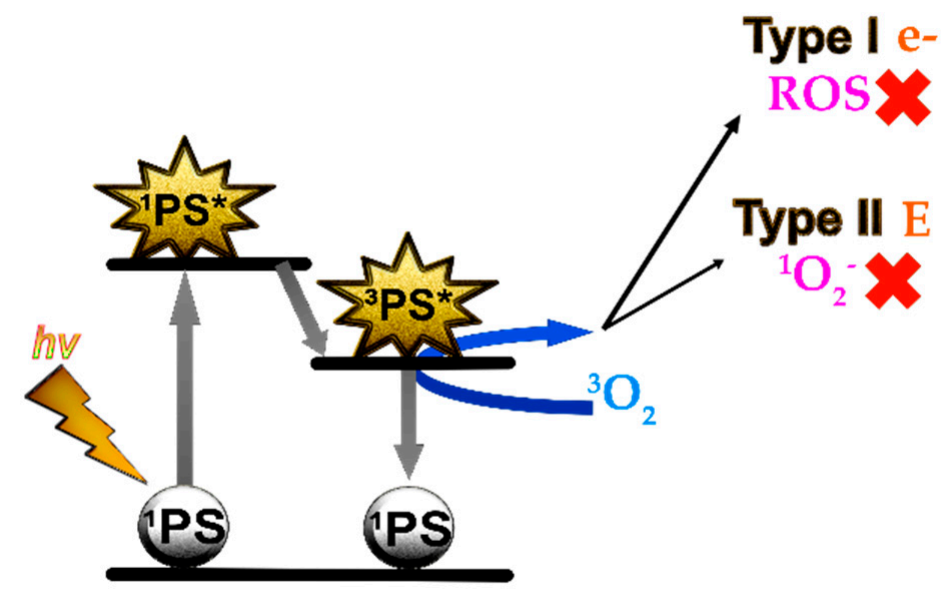

Figure 1. Mechanism of action of antimicrobial photodynamic therapy: photosensitizer molecules (PS) in ground state ( $\left.{ }^{1} \mathrm{PS}\right)$ are activated by light $(h v)$ of a wavelength that matches the absorption wavelength of the molecule. PS reaches an excited state, first singlet $\left({ }^{1}{ }^{P} S^{*}\right)$, very unstable, and rapidly triplet $\left({ }^{3} \mathrm{PS}^{*}\right)$. This reacts with the oxygen by transferring electrons (Type I) or energy (Type II) in its reversion to the ground state $\left({ }^{1}{ }^{P} S^{*}\right)$. Type I reaction is characterized by the formation of reactive oxygen species (ROS) and Type II by the formation of singlet oxygen $\left({ }^{1} \mathrm{O}_{2}{ }^{-}\right)$. These molecules are highly reactive and are capable of damaging cells resulting in a toxic effect for microorganisms (Adapted with permission from ref. [18], 2018, Pérez-Laguna et al.).

Among the advantages of the aPDT is the photoinactivation of the microorganisms independently of their pattern of AMR, without its use contributing to the selection of drug-resistant strains, and its broad spectrum of activity; therefore it does not require a precise microbial diagnosis and it is very useful for infections caused by several microorganisms $[19,20]$. By contrast, the most import limitation is the possibility of microbial regrowth by those who have not been inactivated during irradiation $[17,18,21]$. The combination of aPDT with antibiotic or antifungal treatment is a promising approach to the fight against infectious diseases due to aPDT's ability to increase the susceptibility of microorganisms to antimicrobial drugs and minimize the possibility of the regrowth after aPDT [22-24]. 


\subsection{Objective}

We present a review of articles related to in vitro activities of aPDT in combination with antibiotics or antifungals, focusing on microorganisms that cause SSTIs. The aim is to combine the information that exists about each microorganism (gather the bases of knowledge). This review serves as a starting point for new combined treatment research (expand and apply knowledge).

The questions that are intended to be answered are: (1) Which microorganisms that cause SSTIs have been exposed in vitro to combinations of aPDT and antimicrobials? (2) Which methodologies have been used? (3) Are there an additive or synergistic antimicrobial effect? (4) Which are the best combinations clinically and microbiologically?

\section{Methodology}

\subsection{Eligibility Criteria}

We have considered in vitro studies that used antimicrobial agents (antibiotics or antifungals) plus aPDT against microorganisms that cause SSTIs. The specific requirements for inclusion of the studies were (1) in vitro studies in planktonic state or forming biofilm and ex vivo studies on the skin or mucous membrane; (2) aimed to inactivate identified bacteria, or fungi that cause SSTIs; (3) used antibiotics or antifungals as a fundamental part of the treatment; (4) used aPDT as a fundamental part of the treatment; and (5) published in indexed journals and written in English or Spanish.

\subsection{Study Selection, Data Collection Process, and Characteristics}

The keywords used for the search in Pubmed and Embase library databases were: photodynamic therapy, PDT, antimicrobial photodynamic therapy, aPDT, photodynamic antimicrobial chemotherapy, PACT, photoinactivation, photodynamic inactivation, PDI, combination, combined treatment, antimicrobial agents, antibiotics, and antifungals. The last search was carried out on 1 June 2021 and no time limits in the past were applied. Research papers that meet the eligibility criteria (Section 2.1) were included. A huge number of studies contained the keywords; nevertheless, after applying the eligibility criteria, the number was drastically reduced to a total of 33 .

The data recapitulated were: (1) etiological agent of SSTIs; (2) type of study: in vitro (planktonic or forming biofilm) and ex vivo; (3) antimicrobial methodology: antibiotics or antifungals used and their application and concentration; (4) aPDT methodology: PS used and parameters of irradiation (source type, wavelength, intensity, and fluence); (5) effect of combined treatment against microorganism.

The 33 included studies were grouped depending on the etiological agent of SSTIs. The structure used is as follows:

-Gram-positive bacteria: Staphylococcus spp. (Section 3.1: Section 3.1.1. S. aureus and Section 3.1.2. Staphylococcus epidermidis and Staphylococcus hemolytic)

-Mycobacteria: Mycobacterium fortuitum (Section 3.2.)

-Gram-negative bacteria: E. coli, P. aeruginosa, and A. baumannii (Section 3.3, Section 3.4, Section 3.5 respectively)

-Fungal infectious agents: Yeast (Section 3.6. Candida spp.), dermatophytes, and molds (Section 3.7).

\section{Results of Studies on In Vitro aPDT Combined with Antimicrobial Agents against Infectious Microorganism of Skin and Soft Tissues}

3.1. Staphylococcus spp.

3.1.1. Staphylococcus aureus

S. aureus, frequently involved in the problem of AMR, is the main causative agent of SSTI and represents one of the most important pathogens involved in CSSTI $[2,25,26]$. For this reason, this is the bacteria with more studies trying the combination of different antibiotics and PS published so far. Table 5 summarizes the studies concerning in vitro aPDT plus other antimicrobial treatments against $S$. aureus. 


\section{Porphycene and Porphyrin Studies}

To our knowledge, one of the most recent articles about aPDT and antimicrobials' combination was published by Nieves et al. They report the synthesis of a new porphycene namely 2-aminothiazolo[4,5-c]-2,7,12,17-tetrakis(methoxyethyl)porphycene (ATAZTMPo)gentamicin conjugate. It outperforms the antimicrobial effect against $S$. aureus and E. coli of the two components delivered separately. This novel photoantimicrobial agent may be used to enhance the therapeutic index of gentamicin, broaden the spectrum of pathogens against which it is effective, and reduce its side effects [27].

The combination of gentamicin with porphyrins also seems to be effective against S. aureus $[26,28]$.

5-aminolevulinic acid (5-ALA), a pro-drug that, once metabolized by proliferating bacteria, is converted into the natural PS protoporphyrin IX (PpIX), combined with a low concentration of gentamycin $(2 \mu \mathrm{g} / \mathrm{mL})$ improved qualitative and quantitative antibacterial effects against $S$. aureus biofilms. The proposed explanation was that photoactivation generated ROS which damages or kills the cells, while gentamicin, even at low doses, completes the eradication. The mechanism of action of gentamicin is based on its capacity to bind tightly to the $30 \mathrm{~S}$ subunit of the bacterial ribosome impairing protein synthesis and the bacterial cells die. The fundamental requirement for gentamicin to act as an antibiotic is the need to penetrate the membrane of the bacterial cell, and aPDT can possibly damage the membrane thus increasing antibiotic penetration [28].

Deuteroporphyrin-aPDT combined with different antibiotics included gentamicin, vancomycin, rifampicin, fusidic acid, and oxacillin did not detect synergistic effect except for oxacillin. They concluded that only the combination of deuteroporphyrin-aPDT plus oxacillin had potential for aPDT to improve traditional antibiotic treatment with cell wall synthesis inhibitors [29].

Regarding the combination of porphyrins with other antibiotics different from gentamycin, when ALA-aPDT was combined with netilmicin, vancomycin or cefaclor against S. aureus biofilms, synergistic bactericidal effect was observed. Destruction occurred predominantly in the upper layer of the biofilm, and in a strain-dependent manner. That is why they suggested that a drug sensitivity test should be performed in advance [30].

Meso-tetrakis( $N$-methyl-4-pyridyl) porphine tetra-tosylate (TMP), a cationic porphyrin combined with vancomycin, was highly effective against $S$. aureus biofilms. It seems that the combination blocks cell wall synthesis, and the damaged biofilms may be more susceptible to host defenses which could be useful for biofilms adhering to medical implant surfaces [31].

In contrast, the combination of other cationic porphyrin, 5,10,15,20-tetrakis[4-(3-N,Ndimethylaminopropoxy) phenyl] porphyrin (TAPP)-aPDT with the cell wall inhibiting antibiotics ceftriaxone or vancomycin did not significantly increase the aPDT effect on planktonic S. aureus. However, the bactericidal effect of TAPP-aPDT was additive with the protein synthesis inhibitors chloramphenicol and tobramycin against $S$. aureus and $E$. coli, and was synergistic against methicillin resistant S. aureus (MRSA) and S. epidermidis. It seems that vancomycin and ceftriaxone presented limited effects when combined with TAPP-aPDT; nevertheless, tobramycin and chloramphenicol reached additive effects for S. aureus and E. coli and synergy against MRSA and S. epidermidis, even when sub-minimum inhibitory concentration (MIC) levels of TAPP and antibiotics were used. The results suggest that even sub-MIC levels of photo-activated TAPP could be used to boost the activity of waning antibiotics [32].

Tetracationic porphyrin meso-tetrakis(1-methylpyridinium)porphyrin $\left(\mathrm{Tetra}-\mathrm{Py}{ }^{+}-\mathrm{Me}\right)$ PDT combined with ampicillin significantly reduced $S$ aureus survival in both states (planktonic and biofilm) [33].

Finally, the endogenous porphyrins accumulated by some microorganism, such as E. coli or S. aureus, combined with different wavelengths of light (blue, $470 \mathrm{~nm}$, or red, $625 \mathrm{~nm}$ ) in the presence of ciprofloxacin were more effective than the antibiotic alone [34]. 
Overall, there are multiple promising combinations of antibiotics and porphyrinsPDT which could increase the efficacy in vitro of conventional antibiotics against $S$. aureus (See Table 5).

\section{Phenothiazine Studies}

Another chemical group of PS for PDT are phenothiazinium dyes, such as methylene blue (MB) and toluidine blue $\mathrm{O}(\mathrm{TBO})$.

MB-aPDT combined with chloramphenicol showed an additive effect against planktonic $S$. aureus [32], while ciprofloxacin plus MB-aPDT was synergistic either in planktonic and in biofilm forms [35]. As usual, bacteria forming biofilms were less affected than bacteria in planktonic phase and a bigger effect was seen when ciprofloxacin was applied after aPDT rather than before or simultaneously. Kashef et al. using MB or TBO-aPDT with linezolid against biofilm resulted in a greater reduction in the viable count of bacteria than either of them separately. TBO-aPDT plus linezolid gave the greatest reduction (although the killing was only $<3 \log _{10}$ reduction in viable count for any of the strains) [36].

TBO-aPDT combined with gentamicin and incubated 45-min prior to irradiation showed positive effects against $S$. aureus strains in planktonic state, both in a reference strain and in a multidrug resistant (MDR) clinical isolate; they showed that TBO-aPDT treatment permeabilized the bacterial membranes, promoted gentamicin cellular accumulation and the intracellular ROS generation by the combination was much higher than that of single treatment groups [37].

Our group explored the effect of MB-aPDT, alone or in combination with gentamicin, against planktonic and biofilm S. aureus. The addition of gentamicin (concentrations that by themselves do not achieve any effect) caused the complete photoelimination in the case of planktonic $S$. aureus, with a lower MB-PDT dose, whereas it did not produce any change in biofilm [38]. In a previous study, it was proven that concentrations of linezolid or mupirocin which did not harm the bacteria also exert a synergistic effect when they were combined with MB, also reducing the concentration of the PS needed to photoinactivate planktonic S. aureus [39]. However, recently we have verified in a superficial abrasion mouse model of S. aureus skin infection that the addition of mupirocin to MB-aPDT treatment improved antimicrobial activity but it did not enhance wound healing [40].

Xanthene Studies

Rose Bengal (RB, a xanthene dye) has been rehearsed combined with mupirocin, linezolid, or gentamicin with a synergistic bactericidal effect in planktonic S. aureus [39]. Additionally, the combination of RB-aPDT plus gentamicin was evaluated against $S$. aureus biofilms. However, only high concentration of RB $(64 \mu \mathrm{g} / \mathrm{mL})$ and gentamicin $(40 \mu \mathrm{g} / \mathrm{mL})$ showed a synergistic effect against biofilms [41]. It is interesting that the combination of RB-aPDT with methicillin significantly reduced the MIC of methicillin either of MRSA or methicillin sensitive $S$. aureus [26].

\subsubsection{Staphylococcus epidermidis and Staphylococcus haemolyticus}

S. epidermidis and S. haemolyticus are part of the skin flora of humans but in specific circumstances, such as with immunocompromised or hospitalized patients with catheters or other surgical implants, they are well-known opportunistic pathogens which cause local or systemic infections. The highly antibiotic-resistant phenotype and their ability to form biofilms makes infections difficult to treat [42-45].

The study of Barra et al. has explored 5-ALA-aPDT plus gentamicin at a low concentration against clinical isolates of S. haemolyticus, S. epidermidis, and S. aureus biofilms; they reported a synergistic effect being $S$. haemolyticus the most sensitive to photoinactivation [28].

Other porphyrins, such as TMP-aPDT combined with vancomycin in biofilms [46] or TAPP-aPDT combined with chloramphenicol or tobramycin in planktonic cells, showed a higher efficacy against S. epidermidis than any of them separately. The authors hypothesized 
that TAPP could act in combination with lower concentrations of antibiotics providing a controlled release of the antibiotic, and in consequence improving their efficacy to maintain an antimicrobial effect [32].

In conclusion, multiple antibiotics in combination with different types of PDT based on protoporphyrins showed improved bactericidal effects against planktonic S. epidermidis or forming biofilms $[28,32,46]$.

Table 5 includes a summary of all the studies of aPDT combined with antibiotics. Table 2 summarizes in detail the methodology and the results of different studies that reported the in vitro activity of aPDT plus antibiotics against S. haemolyticus and S. epidermidis (Table 2: Biofilm Studies and biofilm state, planktonic_Table 2: Planktonic Cell Studies).

Table 2. Studies combining in vitro aPDT plus other treatment against S. haemolyticus and S. epidermidis comparing the methodology and the results biofilm studies, planktonic cell studies.

\begin{tabular}{|c|c|c|c|c|c|c|c|c|c|c|c|}
\hline \multicolumn{12}{|c|}{ Biofilm Ttudies } \\
\hline Target & PS & $\begin{array}{c}\text { PS } \\
\text { Concen- } \\
\text { tration } \\
(\mu \mathrm{M})\end{array}$ & Antibiotic & $\begin{array}{c}\text { Antibiotic } \\
\text { Concen- } \\
\text { tration } \\
(\mu \mathrm{g} / \mathrm{mL})\end{array}$ & $\begin{array}{l}\text { Source } \\
\text { Type }\end{array}$ & $\begin{array}{l}\text { Wavelength } \\
\text { (nm) }\end{array}$ & $\begin{array}{l}\text { Intensity } \\
\left(\mathrm{mw} / \mathrm{cm}^{2}\right)\end{array}$ & $\begin{array}{l}\text { Fluence } \\
\left(\mathrm{J} / \mathrm{cm}^{2}\right)\end{array}$ & $\begin{array}{l}\text { Inactivation } \\
\text { Fraction (\%) }\end{array}$ & $\begin{array}{l}\text { Synergistic } \\
\text { Observed } \\
\text { Effect }\left({ }^{*}\right)\end{array}$ & Reference \\
\hline $\begin{array}{c}\text { S. } \\
\text { haemolyti- } \\
\text { cus } \\
\text { clinical } \\
\text { isolate }\end{array}$ & 5-ALA & 40 & gentamicin & 2 & 50-LED & $630 \pm 15$ & 83 & 250 & $\sim 70$ & $>$ inactivation & [28] \\
\hline $\begin{array}{l}\text { S. epider- } \\
\text { midis } \\
\text { clinical } \\
\text { isolate }\end{array}$ & 5-ALA & 40 & gentamicin & 2 & 50-LED & $630 \pm 15$ & 83 & 250 & $\sim 75$ & >inactivation & [28] \\
\hline $\begin{array}{l}\text { S. epider- } \\
\text { midis } \\
\text { RP62A \& } \\
5179 R\end{array}$ & TMP & 10 & vancomycin & 200 & $\begin{array}{l}\text { tungsten } \\
\text { lamp }\end{array}$ & $400-800$ & 166 & 300 & 99.9999 & >inactivation & [46] \\
\hline \multicolumn{12}{|c|}{ Planktonic Cell Studies } \\
\hline Target & PS & $\begin{array}{c}\text { PS } \\
\text { Concen- } \\
\text { tration } \\
(\mu \mathrm{M})\end{array}$ & Antibiotic & $\begin{array}{c}\text { Antibiotic } \\
\text { Concen- } \\
\text { tration } \\
(\mathrm{mg} / \mathrm{L})\end{array}$ & $\begin{array}{l}\text { Preincuba } \\
\text { tion Time } \\
\text { (h) }\end{array}$ & $\begin{array}{l}\text { Irradiation } \\
\text { Time (h) }\end{array}$ & $\begin{array}{l}\text { Source } \\
\text { Type }\end{array}$ & $\begin{array}{l}\text { Wave } \\
\text { length }\end{array}$ & Media & $\underset{\text { Well }}{\mathrm{CFU} / 200} \mu \mathrm{L}$ & $\begin{array}{c}\log _{10} \operatorname{Re}- \\
\text { duction }\end{array}$ \\
\hline $\begin{array}{l}\text { S. epider- } \\
\text { midis } \\
\text { ATCC } \\
35984\end{array}$ & TAPP & 5 & $\begin{array}{l}\text { chloram } \\
\text { phenicol }\end{array}$ & 2 & 19 & 5 & $\begin{array}{l}100 \mathrm{~W}, \\
120 \mathrm{~V} \\
\text { Sylvania } \\
\text { white } \\
\text { light }\end{array}$ & $\begin{array}{l}\text { Broad } \\
\text { spec- } \\
\text { trum }\end{array}$ & TSB & $\sim 10^{6}$ & $\sim 2$ \\
\hline $\begin{array}{l}\text { S. epider- } \\
\text { midis } \\
\text { ATCC } \\
35984\end{array}$ & TAPP & 5 & tobramycin & 4.5 & 19 & 5 & $\begin{array}{c}100 \mathrm{~W}, \\
120 \mathrm{~V} \\
\text { Sylvania } \\
\text { white } \\
\text { light }\end{array}$ & $\begin{array}{l}\text { Broad } \\
\text { spec- } \\
\text { trum }\end{array}$ & TSB & $\sim 10^{6}$ & $\sim 3$ \\
\hline
\end{tabular}

$(*)$ : the combination causes an increase in percentage of microbial inactivation greater than the sum of the antibacterial activity of the aPDT plus the antibiotic treatment when they are applied alone; 5-ALA: 5-aminolevulinic acid; aPDT: antimicrobial photodynamic therapy; CFU: colony forming unit; LED: light-emitting diode; PS: photosensitizer; TAPP: meso-tetra (4-aminophenyl) porphine; TMP: tetra-substituted N-methyl-pyridyl-porphine; TSB: trypticase soy broth.

\subsection{Mycobacterium fortuitum}

M. fortuitum is an atypical mycobacteria involved in skin infections very difficult to treat, thus usually causes refractory infections $[47,48]$.

The combination of MB-aPDT with ciprofloxacin, moxifloxacin, or amikacin against a clinical isolate had in vitro synergistic antimicrobial effect (the latter also was tested in vivo to treat a model of keratitis in rabbits resulting in significantly less bacterial burden). Sublethal concentrations of antibiotic plus sublethal dosage of MB reached a mycobacterial survival reduction in the colony forming units (CFU) of at least $2 \log _{10}$ lesser compared to the reduction achieved with antibiotics alone, although this effect became insignificant when higher doses of antibiotics were used. The maximum degree of inactivation was achieved by the combination of MB-aPDT with ciprofloxacin or moxifloxacin $\left(\geq 4 \log _{10}\right.$ reduction, with amikacin $\left(\sim 3 \log _{10}\right.$ reduction) [49]. Table 5 and Table 3 summarize the methodology and the results of this study. 
Table 3. Comparison of methodology and results of an in vitro study of aPDT followed by culture with antibiotics on Mycobacterium fortuitum.

\begin{tabular}{|c|c|c|c|c|c|c|c|c|c|c|c|c|c|}
\hline Strain & PS & $\begin{array}{c}\text { PS } \\
\text { Concen- } \\
\text { tration } \\
(\mathrm{mg} / \mathrm{mL})\end{array}$ & Antibiotic & $\begin{array}{c}\text { Antibiotic } \\
\text { Concentra- } \\
\text { tion } \\
(\mathrm{mg} / \mathrm{mL})\end{array}$ & $\begin{array}{c}\text { Preincu } \\
\text { bation } \\
\text { Time (h) }\end{array}$ & $\begin{array}{l}\text { Source } \\
\text { Type }\end{array}$ & $\begin{array}{c}\text { Wave } \\
\text { length } \\
(\mathrm{nm})\end{array}$ & $\begin{array}{l}\text { Intensity } \\
\left(\mathrm{mw} / \mathrm{cm}^{2}\right)\end{array}$ & $\begin{array}{l}\text { Fluence } \\
\left(\mathrm{J} / \mathrm{cm}^{2}\right)\end{array}$ & $\begin{array}{l}\text { Media/ } \\
\text { Culture }\end{array}$ & $\begin{array}{c}\text { CFU/ } \\
200 \mu \mathrm{L} \\
\text { Well }\end{array}$ & $\begin{array}{l}\text { Synergistic } \\
\text { Observed } \\
\text { Effect }\left(^{*}\right)\end{array}$ & Reference \\
\hline \multirow{3}{*}{$\begin{array}{c}\text { M. } \\
\text { fortuitum } \\
\text { clinical } \\
\text { isolate }\end{array}$} & \multirow{3}{*}{ MB } & \multirow{3}{*}{50} & amikacin & $0-0.5$ & \multirow{3}{*}{$\begin{array}{l}0+72 \\
\text { with } \\
\text { antib }\end{array}$} & \multirow{3}{*}{$\begin{array}{c}\text { metal } \\
\text { halogen } \\
\text { lamp }\end{array}$} & \multirow{3}{*}{$560-780$} & \multirow{3}{*}{100} & \multirow{3}{*}{100} & PBS & \multirow{3}{*}{$\sim 10^{8}$} & \multirow{3}{*}{$\begin{array}{l}>\text { inactivation } \\
\left(\geq 2 \log _{10}\right. \\
\text { reduction) }\end{array}$} & \multirow{3}{*}{ [49] } \\
\hline & & & $\begin{array}{l}\text { ciprofloxacin } \\
\text { hydrochloride }\end{array}$ & $0-0.06$ & & & & & & $\begin{array}{l}\text { with } \\
0.02 \% \\
\text { Tween }\end{array}$ & & & \\
\hline & & & $\begin{array}{l}\text { moxifloxacin } \\
\text { hydrochloride }\end{array}$ & $0-0.06$ & & & & & & $\begin{array}{l}\text { Muller } \\
\text { Hilton }\end{array}$ & & & \\
\hline
\end{tabular}

$\left({ }^{*}\right)$ : the combination causes an increase in percentage of microbial inactivation greater than the sum of the antibacterial activity of the aPDT plus the antibiotic treatment when they are applied alone; aPDT: antimicrobial photodynamic therapy; MB: methylene blue; PBS: phosphate-buffered solution.

\subsection{Escherichia coli}

E. coli is the best-known bacteria of the Enterobacteriaceae family [50]. Even though some serotypes of $E$. coli can cause serious disease, most strains are harmless and can only act as opportunistic pathogens [51].

Table 5 includes a summary of the in vitro aPDT plus antibiotics against E. coli studies and Table 4 summarizes the methodology and the results of Gram-negatives studies.

Table 4. Studies on combination of in vitro aPDT plus other treatment against Gram-negative bacteria that cause SSTIs comparing the methodology and the results.

\begin{tabular}{|c|c|c|c|c|c|c|c|c|c|c|}
\hline Strain & PS & Antibiotic & Phase & Source Type & $\begin{array}{c}\text { Wavelength } \\
\text { (nm) }\end{array}$ & $\begin{array}{l}\text { Intensity } \\
\left(\mathrm{mw} / \mathrm{cm}^{2}\right)\end{array}$ & $\begin{array}{l}\text { Fluence } \\
\left(\mathrm{J} / \mathrm{cm}^{2}\right)\end{array}$ & Synergy & $\begin{array}{c}\text { Observed } \\
\text { Effect }(*)\end{array}$ & Reference \\
\hline $\begin{array}{c}\text { E. coli ATCC } \\
25922\end{array}$ & ATAZTMPo & gentamicin & planktonic & $\begin{array}{l}\text { LED (Sorisa } \\
\text { Photocare) }\end{array}$ & $638 \pm 9 \mathrm{~nm}$ & 17 & 45 & yes & >inactivation & [27] \\
\hline $\begin{array}{l}\text { E. coli ATCC } \\
25922\end{array}$ & TAPP & $\begin{array}{l}\text { tobramycin } \\
\text { or chloram- } \\
\text { phenicol }\end{array}$ & planktonic & $\begin{array}{c}100 \mathrm{~W}, 120 \mathrm{~V} \\
\text { Sylvania } \\
\text { white light }\end{array}$ & $\begin{array}{l}\text { Broad } \\
\text { spectrum }\end{array}$ & ND & ND & no & additivity & [32] \\
\hline $\begin{array}{l}\text { E. coli ATCC } \\
25922\end{array}$ & $\mathrm{MB}$ & ciprofloxacin & planktonic & $\begin{array}{c}\text { IrradLED }^{\circledR} \\
\text { biopdi, São } \\
\text { Carlos, SP, } \\
\text { Brazil }\end{array}$ & $\sim 660$ & ND & $\begin{array}{c}2.8 \text { and } \\
5.6\end{array}$ & yes & >inactivation & [35] \\
\hline $\begin{array}{l}\text { E. coli ATCC } \\
25922\end{array}$ & MB & ciprofloxacin & biofilm & $\begin{array}{c}\text { IrradLED }^{\circledR} \\
\text { biopdi, São } \\
\text { Carlos, SP, } \\
\text { Brazil }\end{array}$ & 660 & ND & $\begin{array}{l}11.2 \text { and } \\
22\end{array}$ & yes & >inactivation & [35] \\
\hline $\begin{array}{l}\text { E. coli ATCC } \\
9027 \text { and } \\
\text { MDR clinical } \\
\text { isolates }\end{array}$ & $\begin{array}{l}\text { endogenous } \\
\text { porphyrins }\end{array}$ & $\begin{array}{c}\text { ciprofloxacin } \\
\text { or } \\
\text { norfloxacin } n\end{array}$ & planktonic & $\begin{array}{c}\text { LED } \\
\text { Dermaled }^{\circledR}\end{array}$ & $\begin{array}{l}\sim 470 \text { and } \\
\quad \sim 625\end{array}$ & ND & ND & yes & $\begin{array}{l}\text { incrase in } \\
\text { halo }\end{array}$ & [34] \\
\hline E. coli & Chlorin e6 & $\begin{array}{c}\text { colistin, } \\
\text { ciprofloxacin } \\
\text { or amikacin }\end{array}$ & planktonic & $\begin{array}{c}\text { diode laser, } \\
\text { Laser } \\
\text { Coupler } 635 \\
\text { (Wroclaw, } \\
\text { Poland) }\end{array}$ & 635 & 290 & 120 & yes & >inactivation & [51] \\
\hline E. coli & $\begin{array}{c}\text { Tetra-Py }{ }^{+}- \\
\text {Me }\end{array}$ & $\begin{array}{l}\text { ampicillin or } \\
\text { chloram- } \\
\text { phenicol }\end{array}$ & planktonic & $\begin{array}{l}\text { white light } \\
\text { lamps (13 } \\
\text { lamps } \\
\text { OSRAM } 21 \text { of } \\
\text { 18 W each) }\end{array}$ & $\begin{array}{c}\text { Broad } \\
\text { spectrum } \\
380 \text { to } 700\end{array}$ & 40 & - & yes & >inactivation & [52] \\
\hline $\begin{array}{c}\text { E. coli, E. } \\
\text { aerogenes, and } \\
\text { K. pneumoniae } \\
\text { resistant to } \\
3^{\text {rd_- }} \\
\text { cephalosporins, } \\
\text { clinical } \\
\text { isolates }\end{array}$ & MB & ceftriaxone & planktonic & $\begin{array}{c}\text { LED } \\
\text { (Biopdi/Irrad- } \\
\text { Led } \\
660)\end{array}$ & $660 \mathrm{n} \pm 5$ & 25 & 25 & no & indifference & [53] \\
\hline $\begin{array}{l}\text { P. aeruginosa } \\
\text { PAO1 }\end{array}$ & TMP & tobramycin & biofilm & $\begin{array}{c}\text { mercury } \\
\text { vapor lamp }\end{array}$ & $\begin{array}{c}\text { Broad } \\
\text { spectrum }\end{array}$ & - & $220-240$ & yes & $\begin{array}{c}\text { >inactivation } \\
\& \\
\text { tobramycin } \\
\text { MIC } \\
\text { decreased }\end{array}$ & [54] \\
\hline
\end{tabular}


Table 4. Cont.

\begin{tabular}{|c|c|c|c|c|c|c|c|c|c|c|}
\hline Strain & PS & Antibiotic & Phase & Source Type & $\begin{array}{c}\text { Wavelength } \\
\text { (nm) }\end{array}$ & $\begin{array}{l}\text { Intensity } \\
\left(\mathrm{mw} / \mathrm{cm}^{2}\right)\end{array}$ & $\begin{array}{l}\text { Fluence } \\
\left(\mathrm{J} / \mathrm{cm}^{2}\right)\end{array}$ & Synergy & $\begin{array}{l}\text { Observed } \\
\text { Effect }\left(^{*}\right)\end{array}$ & Reference \\
\hline $\begin{array}{l}\text { P. aeruginosa } \\
\text { PAO1 }\end{array}$ & $\begin{array}{l}\text { endogenous } \\
\text { porphyrins }\end{array}$ & gentamicin & planktonic & $\begin{array}{l}\text { Nd:YAG laser } \\
\text { continuous / } \\
\text { Pulsed-Q } \\
\text { switched }\end{array}$ & 532 & 106 & & yes & >inactivation & [55] \\
\hline $\begin{array}{l}\text { P. aeruginosa } \\
\text { PAOland } \\
\text { others MDR } \\
\text { and XDR }\end{array}$ & $\begin{array}{l}\text { endogenous } \\
\text { porphyrins }\end{array}$ & $\begin{array}{l}\text { gentamicin, } \\
\text { meropenem } \\
\text { or } \\
\text { ceftazidime }\end{array}$ & planktonic & $\begin{array}{l}\text { Single- } \\
\text { emitter diode } \\
\text { lamp }\end{array}$ & 410 & 15.7 & 50 & yes & $\begin{array}{c}\text { >inactivation } \\
\& \\
\text { antibiotic } \\
\text { MIC } \\
\text { decreased }\end{array}$ & [56] \\
\hline $\begin{array}{c}\text { ATCC } \\
27853 \\
\text { P. aeruginosa } \\
\text { ATCC } 27853\end{array}$ & MB & ofloxacin & planktonic & LED & $\sim 637$ & 44 & & yes & >inactivation & [57] \\
\hline $\begin{array}{l}\text { P. aeruginosa } \\
\text { ATCC } 27853\end{array}$ & MB & gentamicin & planktonic & $\begin{array}{l}\text { LED lamp } \\
\text { (Showtec } \\
\text { LED Par 64 } \\
\text { Short 18 x } \\
\text { RGB 3-in-1 } \\
\text { LED, Highlite } \\
\text { International } \\
\text { B.V. Spain) }\end{array}$ & $625 \pm 10$ & 7 & 18 & yes & $\begin{array}{l}\text { bactericidal } \\
\text { effect with } \\
\text { lower } \\
\text { MB-PDT } \\
\text { dose }\end{array}$ & [38] \\
\hline $\begin{array}{l}\text { P. aeruginosa } \\
\text { ATCC } 27853\end{array}$ & MB & gentamicin & biofilms & $\begin{array}{l}\text { LED lamp } \\
\text { (Showtec } \\
\text { LED Par } 64 \\
\text { Short } 18 x \\
\text { RGB 3-in-1 } \\
\text { LED, Highlite } \\
\text { International } \\
\text { B.V. Spain) }\end{array}$ & $625 \pm 10$ & 7 & 18 & yes & $\begin{array}{l}\text { bactericidal } \\
\text { effect with } \\
\text { lower } \\
\text { MB-PDT } \\
\text { dose }\end{array}$ & [38] \\
\hline $\begin{array}{l}\text { P. aeruginosa } \\
\text { ATCC } 10145 \\
\text { and } 35 \\
\text { clinical } \\
\text { isolates } \\
\text { including } \\
\text { MDR and } \\
\text { XDR }\end{array}$ & $\mathrm{RB}$ & $\begin{array}{l}\text { camel or } \\
\text { pexiganan }\end{array}$ & planktonic & $\begin{array}{c}\text { LED lamps } \\
\text { (SecureMe- } \\
\text { dia, } \\
\text { Poland) }\end{array}$ & $\sim 514$ & 23 & 60 & yes & >inactivation & [58] \\
\hline $\begin{array}{l}\text { P. aeruginosa } \\
\text { ATCC } 25668 \\
\text { and sensitive } \\
\text { and resistant } \\
\text { clinical } \\
\text { isolates }\end{array}$ & $\mathrm{RB}$ & sulfanilamide & planktonic & $\begin{array}{l}18 \mathrm{~W} \text { white } \\
\text { luminescent } \\
\text { lamp }\end{array}$ & $\begin{array}{l}\text { Broad } \\
\text { spectrum } \\
400-700\end{array}$ & 1.25 & - & no & indifference & [26] \\
\hline $\begin{array}{l}\text { A. baumannii } \\
2 \text { XDR } \\
\text { clinical } \\
\text { isolates }\end{array}$ & $\mathrm{RB}$ & $\begin{array}{c}\text { gentamycin, } \\
\text { doxycicline, } \\
\text { trimethoprim- } \\
\text { sulfamethoxa } \\
\text { zole, } \\
\text { ciprofloxacin, } \\
\text { imipenem, } \\
\text { piperacillin- } \\
\text { tazobactam, } \\
\text { ceftazidime, } \\
\text { ampicillin- } \\
\text { sulbactam, } \\
\text { colistin }\end{array}$ & planktonic & LED & 515 & 70 & 300 & yes & $\begin{array}{c}\text { >inactivation } \\
\& \\
\text { antibiotic } \\
\text { MIC } \\
\text { decreased }\end{array}$ & [59] \\
\hline $\begin{array}{l}\text { A. baumannii } \\
2 \text { XDR } \\
\text { clinical } \\
\text { isolates }\end{array}$ & $\begin{array}{l}\text { endogenous } \\
\text { porphyrins }\end{array}$ & $\begin{array}{c}\text { gentamycin, } \\
\text { doxycicline, } \\
\text { trimethoprim- } \\
\text { sulfamethoxa } \\
\text { zole, } \\
\text { ciprofloxacin, } \\
\text { imipenem, } \\
\text { piperacillin- } \\
\text { tazobactam, } \\
\text { ceftazidime, } \\
\text { ampicillin- } \\
\text { sulbactam, } \\
\text { colistin }\end{array}$ & planktonic & LED & 411 & 130 & 109.1 & yes & $\begin{array}{c}\text { >inactivation } \\
\& \\
\text { antibiotic } \\
\text { MIC } \\
\text { decreased }\end{array}$ & [59] \\
\hline
\end{tabular}

$\left(^{*}\right)>$ inactivation: the combination causes an increase in the percentage of microbial inactivation greater than the sum of the antibacterial activity of the aPDT plus the antibiotic treatment when they are applied alone; additivity: the combination causes an increase in percentage of microbial inactivation equal to the sum of the antibacterial activity of the aPDT plus the antibiotic treatment when they are applied alone: Indifference was defined as no change from the most active antibiotic treatment; aPDT: antimicrobial photodynamic therapy; ATAZTMPo; 2-aminothiazolo[4,5-c]-2,7,12,17-tetrakis(methoxyethyl)porphycene; RB: rose bengal; LED: light-emitting diode; MB: methylene blue; MDR: multidrug resistant; MIC: minimum inhibitory concentration; ND: no data; PS: photosensitizer; TAPP: meso-tetra (4-aminophenyl) porphine; TBO: toluidine blue O; Tetra-Py+-Me: 5,10,15,20-tetrakis(1-methylpyridinium-4-yl)porphyrin tetra-iodide; TMP: meso-tetra (N-methyl-4-pyridyl) porphine tetra tosylate; XDR: extensively-drug resistant. 
Dastgheyb et al., Ronqui et al., and Nieves et al. investigated not only strains of E. coli but also $S$. aureus. As has been previously commented, results are similar in both species $[27,32,35]$.

\subsubsection{Porphycene Study}

Nieves et al. report the synthesis of a new porphycene, ATAZTMPo -gentamicin conjugate, that is able to reduce $8 \log _{10}$ of surviving planktonic E. coli while it did not exert any effect in darkness. The gentamicin concentration used was close to the reported MIC $(3.8 \mu \mathrm{g} / \mathrm{mL}$ and $4 \mu \mathrm{g} / \mathrm{mL}$ respectively). Nevertheless, MIC values were determined at inoculum sizes 100-1000-fold, more diluted than those used in this study, and the antimicrobial effect usually was evaluated after $24 \mathrm{~h}$ of incubation with the antibiotic, while only $1 \mathrm{~h}$ of preirradiation incubation was allowed in these experiments; therefore, it is not surprising the lack of gentamicin toxicity in darkness [27].

\subsubsection{Phenothiazine and Porphyrin Studies}

Among Dastgheyb et al. and Ronqui et al. studies, the best results against planktonic E. coli were obtained by the later using MB-aPDT followed by ciprofloxacin. The former evaluated TAPP-aPDT associated with tobramycin or chloramphenicol achieving only additive antibacterial effect [32]. The later achieved a reduction of approximately 7 logs of $E$. coli in planktonic and biofilm using MB-aPDT followed by ciprofloxacin (while the reduction against $S$. aureus biofilms was $5.4 \operatorname{logs}$ ). The most remarkable is that the synergistic effect of aPDT plus ciprofloxacin overcame the resistance of biofilm to aPDT. On the other hand, when the antibiotic is applied after aPDT, lower concentrations than the MIC of ciprofloxacin could be used, since the first sub-MIC led to bacterial reduction of both S. aureus and E. coli in planktonic state. They hypothesize that this combination achieves a higher effect in biofilm than in planktonic, and higher in E. coli than in S. aureus, because MB-aPDT works worse on E. coli biofilm and the effect of the addition of antibiotic therapy may be more evident [35]. Pereira et al. studied the effect of ciprofloxacin and norfloxacin against planktonic E. coli, including MDR clinical isolates, when the strains were irradiated, and they observed because of the endogenous porphyrins an increase in the inhibition halo diameter, indicating that these combinations were synergistic [34]. Other combinations, such as ampicillin or chloramphenicol with aPDT using 5,10,15,20-tetrakis(1methylpyridinium-4-yl) porphyrin tetra-iodide, achieved a greater increase in MDR E. coli killing, especially with ampicillin, at sub-inhibitory and inhibitory concentrations [52]. On the other hand, Costa et al. in a comparative study of the effect of MB-aPDT alone or in combination with ceftriaxone against planktonic clinical isolates of Gram-negative bacteria (E. coli, Klebsiella aerogenes-formerly Enterobacter aerogenes_-and Klebsiella pneumoniae resistant to third-generation cephalosporins) concluded that the combination did not cause an effect on bacterial viability greater than that MB-PDT itself [53].

\subsubsection{Chlorophyll Study}

The combination of ciprofloxacin, amikacin, or colistin with aPDT using chlorin e6 improves the antibacterial activity of antibiotics against planktonic E. coli, being the best combination with ciprofloxacin [51].

\subsection{Pseudomonas aeruginosa}

P. aeruginosa is an opportunistic human pathogen especially causing infections in chronic ulcers and burns. Table 5 includes a summary of the in vitro aPDT plus antibiotics against $P$. aeruginosa studies and more information on the different methodologies can be consulted in Table 4.

\subsubsection{Porphyrin Studies}

Sequential treatments of TMP-aPDT followed by tobramycin against $P$. aeruginosa biofilms showed a synergistic effect. The survival bacteria decreased and biofilms pre- 
treated with TMP-aPDT were substantially more sensitive to tobramycin than untreated biofilms [54].

Different studies employed endogenous porphyrins as the PS. The combination of green light (532 nm) and gentamicin resulted in an antibacterial effect against planktonic $P$. aeruginosa [55]. On the other hand, the combination of sublethal doses of blue light $(410 \mathrm{~nm})$ and antibiotics (gentamicin, meropenem, or ceftazidime) reduced the MIC against some planktonic $P$. aeruginosa strains that displayed MDR and extensive drug resistance profiles [56].

\subsubsection{Phenothiazine Studies}

Another study evaluated the in vitro phototoxicity of MB plus ofloxacin against sensitive or resistant $P$. aeruginosa strains that tolerated ofloxacin. MB-aPDT in combination with antibiotic significantly reduced the viability of $P$. aeruginosa strains compared to either one in monotherapy [57]. Recently, our group showed how gentamicin enhances MBaPDT-induced bacterial photinactivation of planktonic and biofilm P. aeruginosa; an in vitro synergistic effect against both states of $P$. aeruginosa was found, therefore the authors hypothesized that this combination could be useful to manage difficult-to-treat SSTI caused by $P$. aeruginosa [38].

\subsubsection{Xanthene Studies}

The use of RB-aPDT combined with CAMEL or pexiganan (antimicrobial peptides) achieves a total elimination of different $P$. aeruginos $a$ clinical isolates, including MDR and XDR strains, in contrast to none or partial reduction when the treatments are applied separately. In addition, they demonstrated that the combination is safe, without harmful effects in human keratinocytes [58]. On other hand, Ilizirov et al. studied the combination of RB-aPDT with sulfanilamide against sensitive and resistant $P$. aeruginosa clinical isolates reporting the absence of additional effect. They hypothesize that it could be because of the very low rates of bacterial envelope damage by RB at sub-MIC, which was not sufficient for enhancing the action of sulfanilamide and they suggest that maybe is better to choose antibiotics which affect the same cell target sites in order to achieve amplification of their activity [26].

\subsection{Acinetobacter baumannii}

A. baumannii is a threatening human pathogen with outstanding capability to acquire AMR [11] although it rarely causes skin infection SSTIs.

Different antibiotics were combined with RB-aPDT or only blue light to excite endogenous PS against extremely drug resistant $A$. baumannii clinical isolates. The final result was the effective eradication. The bactericidal effect of antibiotics was enhanced with sublethal aPDT or blue light addition applied after them. Moreover, they measured the production of ROS and claimed that its increase with the combined treatment could explain the synergistic activity observed. Table 5 includes a summary of this study (and more information can be seen in Table 3) that stands out for covering all antibiotic categories as well as all antimicrobial mechanisms of action and the wide variety of methods used to test synergy (diffusion assays — disk diffusion and E-test, serial dilution methodology-checkerboard assay, and CFU counting- and time kill curves method-postantibiotic effect) [59].

\subsection{Candida spp.}

Candida spp. cause skin and oral and genital mucosa infections and C. albicans is the most relevant one [60].

Table 5 includes a summary of the different combinations studied using in vitro aPDT plus antifungals against yeasts that cause SSTIs; specific details of the methodology of these studies are provided in Table 6.

The effect of MB-aPDT plus fluconazole was evaluated against fluconazole-resistant C. albicans, Candida glabrata, and Candida krusei. A synergistic combination effect against 
the strains of $C$. albicans and C. glabrata, but not against C. krusei, was found. The effect of MB-aPDT alone against $C$. krusei was not statistically significant compared to the effect of its combination with fluconazole [61].

Snell et al. showed that fluconazole did not enhance $C$. albicans killing induced by aPDT using TMP. However, miconazole improved the fungicidal activity of aPDT using either TMP or MB [62].

\subsection{Dermatophytes and Moulds}

Table 5 includes a summary of the combination treatment studies using in vitro aPDT plus other compounds against dermatophytes and molds that cause SSTIs and an extended version can be consulted in Table 6 .

Trichophyton rubrum causes of athlete's foot, fungal infection of nail and ringworm, worldwide athlete's foot, onychomycosis, jock itch, and ringworm. Morton et al. reported that clotrimazole combined with RB-aPDT had the potential to reduce the MIC of the antifungal drug against spores of T. rubrum clinical isolate. This occurred when pretreatment with the antifungal (sublethal dose) was followed by RB-aPDT. When the order of combination was changed, no reduction in the antifungal MIC was observed [63].

Fonsecaea monophora and Fonsecaea pedrosoi are the main causative agents of chromoblastomycosis in Southern China. It is a chronic skin and subcutaneous fungal infection with low cure and high relapse rates. Hu et al. (2015) treated five refractory and complex cases of chromoblastomycosis with 5-ALA-aPDT combined with oral antifungal drugs. The isolates were evaluated for susceptibility to terbinafine, itraconazole, and voriconazole and 5-ALA-aPDT in vitro, revealing sensitivity to the antifungals, with 5-ALA-aPDT altering the cell wall and increasing ROS production. The results showed that there was an unclear synergistic effect of itraconazole plus 5-ALA-aPDT [64]. Altogether, they conclude that 5-ALA-aPDT combined with oral antifungal drugs is a promising method for the treatment of refractory and complex cases of chromoblastomycosis. This idea was also previously defended by the same authors in a previous clinical case of chromoblastomycosis caused by F. monophora that was successfully treated with terbinafine plus 5-ALA-aPDT. This study was also supported by in vitro experiments where they showed the good outcome of 5-ALA-aPDT applied for the inhibition of F. monophora [65].

Exophiala spp. is an ubiquitous fungal species commonly found in soil and plants which causes chromoblastomycosis [66]. On the other hand, Fusarium solani and Fusarium oxysporum are responsible for approximately $60 \%$ and $20 \%$ of the cases of fusariosis, respectively, which is the second most common mold infection in humans after aspergillosis [67]. Gao et al. investigated both planktonic suspensions and biofilms of E. dermatitidis and Fusarium spp. with MB-aPDT combined with standard antifungal treatments (itraconazole, voriconazole, posaconazole, and amphotericin) achieving encouraging results. The pretreatment with MB-aPDT made them more susceptible to antifungals either in planktonic cultures or in biofilms. Therefore, the combination may help to enhance the antifungal susceptibility to overcome problems with drug resistance issues, and has the potential to reduce antifungal drug dosages decreasing their toxicity [15]. According to the authors, this may be due to an increased membrane permeability caused by aPDT, as suggested previously for C. albicans [68]. 
Table 5. In vitro antimicrobial photodynamic therapy plus other treatment studies on infectious microorganism of skin and soft tissues grouped by photosensitizing family.

\begin{tabular}{|c|c|c|c|c|c|c|}
\hline \multicolumn{7}{|c|}{ S. aureus } \\
\hline PS Group & PS & Antibiotic & Phase & Synergy & Observed Effect $\left(^{*}\right)$ & Reference \\
\hline \multirow{11}{*}{$\begin{array}{l}\text { Porphycene } \\
\text { and Porphyrin }\end{array}$} & ATAZTMPo & gentamicin & planktonic & yes & >inactivation & [27] \\
\hline & 5-ALA & gentamicin & biofilm & yes & >inactivation & [28] \\
\hline & $\mathrm{DP}$ & $\begin{array}{l}\text { gentamicin, } \\
\text { vancomycin, } \\
\text { rifampin, } \\
\text { fusidic acid }\end{array}$ & planktonic & no & additivity & [29] \\
\hline & $\mathrm{DP}$ & oxacillin & planktonic & yes & $\begin{array}{l}\text { oxacillin MIC } \\
\text { decreased }\end{array}$ & [29] \\
\hline & 5-ALA & $\begin{array}{c}\text { netilmicin, } \\
\text { cefaclor, } \\
\text { vancomycin }\end{array}$ & biofilm & yes & >inactivation & {$[30]$} \\
\hline & TMP & vancomycin & biofilm & yes & >inactivation & [31] \\
\hline & TAPP & $\begin{array}{l}\text { vancomycin, } \\
\text { ceftriaxone }\end{array}$ & planktonic & no & indifference & {$[32]$} \\
\hline & TAPP & $\begin{array}{l}\text { chloramphenicol, } \\
\text { tobramycin }\end{array}$ & planktonic & yes-no & $\begin{array}{l}\text { >inactivation MRSA; } \\
\text { additive MSSA }\end{array}$ & [32] \\
\hline & Tetra-Py+-Me & ampicillin & planktonic & yes & $\begin{array}{c}\text { faster bactericidal } \\
\text { effect }\end{array}$ & [33] \\
\hline & Tetra-Py+-Me & ampicillin & pork skin (ex vivo) & yes & >inactivation & [33] \\
\hline & (endogenous) & $\begin{array}{l}\text { ciprofloxacin, } \\
\text { norfloxacin }\end{array}$ & planktonic & yes & >inactivation & {$[34]$} \\
\hline \multirow{9}{*}{ Phenothiazines } & $\mathrm{MB}$ & chloramphenicol & planktonic & no & additivity & [32] \\
\hline & $\mathrm{MB}$ & ciprofloxacin & planktonic & yes & >inactivation & [35] \\
\hline & $\mathrm{MB}$ & ciprofloxacin & biofilm & yes & $>$ inactivation & [35] \\
\hline & $\mathrm{TBO}$ & linezolid & biofilm & yes & >inactivation & [35] \\
\hline & $\mathrm{MB}$ & linezolid & biofilm & no & indifference & [35] \\
\hline & TBO & gentamicin & planktonic & yes & >inactivation & [37] \\
\hline & $\mathrm{MB}$ & gentamicin & planktonic & yes & $\begin{array}{l}\text { bactericidal effect with } \\
\text { lower MB-PDT dose }\end{array}$ & [38] \\
\hline & $\mathrm{MB}$ & gentamicin & biofilm & no & $\begin{array}{c}\text { no significant }> \\
\text { inactivation }\end{array}$ & [38] \\
\hline & $\mathrm{MB}$ & $\begin{array}{l}\text { linezolid, } \\
\text { mupirocin }\end{array}$ & planktonic & yes & $\begin{array}{l}\text { bactericidal effect with } \\
\text { lower MB-PDT dose }\end{array}$ & [39] \\
\hline \multirow{4}{*}{ Xanthenes } & $\mathrm{RB}$ & $\begin{array}{l}\text { linezolid, } \\
\text { mupirocin }\end{array}$ & planktonic & yes & $\begin{array}{l}\text { bactericidal effect with } \\
\text { lower RB-PDT dose }\end{array}$ & [39] \\
\hline & $\mathrm{RB}$ & gentamicin & planktonic & yes & $\begin{array}{l}\text { bactericidal effect with } \\
\text { lower RB-PDT dose }\end{array}$ & [41] \\
\hline & $\mathrm{RB}$ & gentamicin & biofilm & yes & $>$ inactivation & [41] \\
\hline & $\mathrm{RB}$ & methicillin & planktonic & yes & $\begin{array}{c}\text { methicillin MIC } \\
\text { decreased }\end{array}$ & [26] \\
\hline
\end{tabular}


Table 5. Cont.

\begin{tabular}{|c|c|c|c|c|c|c|}
\hline \multicolumn{7}{|c|}{ S. haemolyticus } \\
\hline PS Group & PS & Antibiotic & Phase & Synergy & Observed Effect $(*)$ & Reference \\
\hline $\begin{array}{c}\text { Porphycene } \\
\text { and Porphyrin }\end{array}$ & 5-ALA & gentamicin & biofilm & yes & >inactivation & [28] \\
\hline \multicolumn{7}{|c|}{ S. epidermidis } \\
\hline PS group & PS & Antibiotic & Phase & Synergy & Observed effect $(*)$ & Reference \\
\hline \multirow{3}{*}{$\begin{array}{c}\text { Porphycene } \\
\text { and Porphyrin }\end{array}$} & 5-ALA & gentamicin & biofilm & yes & >inactivation & {$[28]$} \\
\hline & TMP & vancomycin & biofilm & yes & >inactivation & [31] \\
\hline & TAPP & $\begin{array}{l}\text { chloramphenicol, } \\
\text { tobramycin }\end{array}$ & planktonic & yes & >inactivation & [32] \\
\hline \multicolumn{7}{|c|}{ M. fortuitum } \\
\hline PS Group & PS & Antibiotic & Phase & Synergy & Observed Effect $(*)$ & Reference \\
\hline Phenothiazines & MB & $\begin{array}{l}\text { ciprofloxacin, } \\
\text { moxifloxacin or } \\
\text { amikacin }\end{array}$ & planktonic & yes & $>$ inactivation & [49] \\
\hline \multicolumn{7}{|c|}{ E. coli } \\
\hline PS Group & PS & Antibiotic & Phase & Synergy & Observed Effect $(*)$ & Reference \\
\hline \multirow{4}{*}{$\begin{array}{l}\text { Porphycene } \\
\text { andPorphyrin }\end{array}$} & ATAZTMPo & gentamicin & planktonic & yes & >inactivation & [27] \\
\hline & TAPP & $\begin{array}{l}\text { tobramycin or } \\
\text { chlorampheni- } \\
\text { col }\end{array}$ & planktonic & no & additivity & [32] \\
\hline & $\begin{array}{l}\text { endogenous } \\
\text { porphyrins }\end{array}$ & $\begin{array}{l}\text { ciprofloxacin or } \\
\text { norfloxacin } n\end{array}$ & planktonic & yes & incrase in halo & {$[34]$} \\
\hline & Tetra-Py ${ }^{+}-\mathrm{Me}$ & $\begin{array}{l}\text { ampicillin or } \\
\text { chlorampheni- } \\
\text { col }\end{array}$ & planktonic & yes & >inactivation & [52] \\
\hline \multirow{3}{*}{ Phenothiazines } & MB & ciprofloxacin & planktonic & yes & >inactivation & [35] \\
\hline & $\mathrm{MB}$ & ciprofloxacin & biofilm & yes & >inactivation & [35] \\
\hline & $\mathrm{MB}$ & ceftriaxone & planktonic & no & indifference & {$[52]$} \\
\hline Chlorophylls & Chlorin e6 & $\begin{array}{l}\text { colistin, } \\
\text { ciprofloxacin or } \\
\text { amikacin }\end{array}$ & planktonic & yes & >inactivation & [51] \\
\hline
\end{tabular}


Table 5. Cont.

\begin{tabular}{|c|c|c|c|c|c|c|}
\hline \multicolumn{7}{|c|}{ P. aeruginosa } \\
\hline PS Group & PS & Antibiotic & Phase & Synergy & Observed Effect $(*)$ & Reference \\
\hline \multirow{3}{*}{$\begin{array}{l}\text { Porphycene } \\
\text { and Porphyrin }\end{array}$} & TMP & tobramycin & biofilm & yes & $\begin{array}{c}\text { >inactivation \& } \\
\text { tobramycin MIC } \\
\text { decreased }\end{array}$ & {$[54]$} \\
\hline & $\begin{array}{l}\text { endogenous } \\
\text { porphyrins }\end{array}$ & gentamicin & planktonic & yes & >inactivation & [55] \\
\hline & $\begin{array}{l}\text { endogenous } \\
\text { porphyrins }\end{array}$ & $\begin{array}{l}\text { gentamicin, } \\
\text { meropenem or } \\
\text { ceftazidime }\end{array}$ & planktonic & yes & $\begin{array}{c}\text { >inactivation \& } \\
\text { antibiotic MIC } \\
\text { decreased }\end{array}$ & [56] \\
\hline \multirow{3}{*}{ Phenothiazines } & $\mathrm{MB}$ & ofloxacin & planktonic & yes & $>$ inactivation & [57] \\
\hline & MB & gentamicin & planktonic & yes & $\begin{array}{l}\text { bactericidal effect with } \\
\text { lower MB-PDT dose }\end{array}$ & [38] \\
\hline & $\mathrm{MB}$ & gentamicin & biofilm & yes & $\begin{array}{l}\text { bactericidal effect with } \\
\text { lower MB-PDT dose }\end{array}$ & [38] \\
\hline \multirow[t]{2}{*}{ Xanthenes } & $\mathrm{RB}$ & $\begin{array}{c}\text { camel or } \\
\text { pexiganan }\end{array}$ & planktonic & yes & >inactivation & [58] \\
\hline & $\mathrm{RB}$ & sulfanilamide & planktonic & no & indifference & [26] \\
\hline \multicolumn{7}{|c|}{ A. baumannii } \\
\hline PS Group & PS & Antibiotic & Phase & Synergy & Observed Effect $(*)$ & Reference \\
\hline Xanthenes & $\mathrm{RB}$ & $\begin{array}{l}\text { gentamycin, } \\
\text { doxycicline, } \\
\text { trimethoprim- } \\
\text { sulfamethoxazole, } \\
\text { ciprofloxacin, } \\
\text { imipenem, } \\
\text { piperacillin- } \\
\text { tazobactam, } \\
\text { ceftazidime, } \\
\text { ampicillin- } \\
\text { sulbactam, } \\
\text { colistin }\end{array}$ & planktonic & yes & $\begin{array}{c}\text { >inactivation \& } \\
\text { antibiotic MIC } \\
\text { decreased }\end{array}$ & [59] \\
\hline $\begin{array}{l}\text { Porphycene } \\
\text { and Porphyrin }\end{array}$ & $\begin{array}{l}\text { endogenous } \\
\text { porphyrins }\end{array}$ & $\begin{array}{l}\text { gentamycin, } \\
\text { doxycicline, } \\
\text { trimethoprim- } \\
\text { sulfamethoxazole, } \\
\text { ciprofloxacin, } \\
\text { imipenem, } \\
\text { piperacillin- } \\
\text { tazobactam, } \\
\text { ceftazidime, } \\
\text { ampicillin- } \\
\text { sulbactam, } \\
\text { colistin }\end{array}$ & planktonic & yes & $\begin{array}{c}\text { >inactivation \& } \\
\text { antibiotic MIC } \\
\text { decreased }\end{array}$ & [59] \\
\hline \multicolumn{7}{|c|}{ Candida spp. } \\
\hline PS Group & PS & Antibiotic & Phase & Synergy & Observed Effect $(*)$ & Reference \\
\hline \multirow{2}{*}{ Phenothiazines } & $\mathrm{MB}$ & fluconazole & planktonic & yes/no & $\geq$ inactivation & [61] \\
\hline & $\mathrm{MB}$ & miconazole & planktonic & yes & >inactivation & {$[62]$} \\
\hline \multirow{2}{*}{$\begin{array}{l}\text { Porphycene } \\
\text { and Porphyrin }\end{array}$} & TMP & miconazole & planktonic & yes & >inactivation & [62] \\
\hline & TMP & fluconazole & planktonic & no & indifference & [62] \\
\hline
\end{tabular}


Table 5. Cont.

\begin{tabular}{|c|c|c|c|c|c|c|}
\hline \multicolumn{7}{|c|}{ Dermatophytes and moulds } \\
\hline PS Group & PS & Antibiotic & Phase & Synergy & Observed Effect $(*)$ & Reference \\
\hline Xanthenes & $\mathrm{RB}$ & clotrimazole & $\begin{array}{l}\text { Planktonic (in vitro: } \\
\text { spores) }\end{array}$ & yes & $\geq$ inactivation & [63] \\
\hline $\begin{array}{l}\text { Porphycene } \\
\text { and Porphyrin }\end{array}$ & 5-ALA & itraconazole & planktonic & yes & $\geq$ inactivation & {$[64]$} \\
\hline \multirow[t]{2}{*}{ Phenothiazines } & $\mathrm{MB}$ & $\begin{array}{l}\text { itraconazole, } \\
\text { voriconazole, } \\
\text { posaconazole, } \\
\text { amphotericin }\end{array}$ & $\begin{array}{l}\text { planktonic and } \\
\text { biofilms }\end{array}$ & yes & MIC decreased & [15] \\
\hline & MB & itraconazole & planktonic & yes & >inactivation & [69] \\
\hline
\end{tabular}

$(*)>$ inactivation: the combination causes an increase in the percentage of microbial inactivation, greater than the sum of the antibacterial activity of the aPDT plus the antibiotic treatment when they are applied alone. Additivity: the combination causes an increase in the percentage of microbial inactivation equal to the sum of the antibacterial activity of the aPDT plus the antibiotic treatment when they are applied alone. Indifference was defined as no change from the most active treatment. 5-ALA $=5$-aminolevulinic acid; ATAZTMPo $=2$-aminothiazolo[4,5c]-2,7,12,17-tetrakis(methoxyethyl)porphycene; $\mathrm{DP}=$ deuteroporphyrin; $\mathrm{MB}=$ methylene blue; $\mathrm{MIC}=$ minimum inhibitory concentration; MRSA = methicillin resistant $S$. aureus; MSSA = methicillin sensitive $S$. aureus; RB = rose bengal; $\mathrm{PS}=$ photosensitizer; TAPP = 5,10,15,20-tetrakis [4-(3-N,N-dimethylaminopropoxy) phenyl] porphyrin; TBO = toluidine blue $\mathrm{O}$; TAPP = meso-tetra (4-aminophenyl) porphine; TMP = tetrasubstituted $\mathrm{N}$-methyl-pyridyl-porphine; Tetra-Py+-Me = 5,10,15,20-tetrakis (1-methylpyridinium-4-yl) porphyrin tetra-iodide.

Table 6. Studies on combination of in vitro aPDT plus other treatment against fungus that cause SSTIs comparing the methodology and the results.

\begin{tabular}{|c|c|c|c|c|c|c|c|c|c|c|}
\hline Strain & PS & Antifungal & Phase & Source Type & $\begin{array}{l}\text { Wavelength } \\
\text { (nm) }\end{array}$ & $\begin{array}{l}\text { Intensity } \\
\left(\mathrm{mw} / \mathrm{cm}^{2}\right)\end{array}$ & $\begin{array}{l}\text { Fluence } \\
\left(\mathrm{J} / \mathrm{cm}^{2}\right)\end{array}$ & Synergy & $\begin{array}{l}\text { Observed } \\
\text { Effect }\left({ }^{*}\right)\end{array}$ & Reference \\
\hline $\begin{array}{l}\text { fluconazole- } \\
\text { resistant } C \text {. } \\
\text { albicans and } C \text {. } \\
\text { glabratai }\end{array}$ & MB & fluconazole & planktonic & InGaAlP LED & nd & 200 & - & yes & >inactivation & [61] \\
\hline $\begin{array}{l}\text { fluconazole- } \\
\text { resistant } C \text {. } \\
\text { krusei }\end{array}$ & MB & fluconazole & planktonic & InGaAlP LED & nd & 200 & - & no & indifference & [61] \\
\hline $\begin{array}{l}\text { C. albicans } \\
\text { SC5314 }\end{array}$ & TMP & miconazole & planktonic & $\begin{array}{c}\text { broadband } \\
\text { visible light } \\
\text { (Sylvania } \\
\text { GRO-LUX, 15 W, } \\
\text { part no. } \\
\text { F15T8/GRO) }\end{array}$ & $575-700$ & 4 & 1 & yes & >inactivation & [62] \\
\hline $\begin{array}{l}\text { C. albicans } \\
\text { SC5314 }\end{array}$ & TMP & fluconazole & planktonic & $\begin{array}{c}\text { broadband } \\
\text { visible light } \\
\text { (Sylvania } \\
\text { GRO-LUX, 15 W, } \\
\text { part no. } \\
\text { F15T8/GRO) }\end{array}$ & $575-700$ & 4 & 1 & no & indifference & [62] \\
\hline $\begin{array}{l}\text { C. albicans } \\
\text { SC5314 }\end{array}$ & MB & miconazole & planktonic & $\begin{array}{c}\text { broadband } \\
\text { visible light } \\
\text { (Sylvania } \\
\text { GRO-LUX, 15 W, } \\
\text { part no. } \\
\text { F15T8/GRO) }\end{array}$ & $575-700$ & 4 & 7.2 & yes & $>$ inactivation & [62] \\
\hline $\begin{array}{l}\text { T. rubrum } \\
\text { clinical isolate }\end{array}$ & $\mathrm{RB}$ & clotrimazole & $\begin{array}{l}\text { Planktonic } \\
\text { (in vitro: } \\
\text { spores) }\end{array}$ & LED & 530 & 13.4 & 12 & yes & $\geq$ inactivation & [63] \\
\hline $\begin{array}{l}\text { F. monophora } \\
\text { clinical } \\
\text { isolates }\end{array}$ & 5-ALA & itraconazole & planktonic & $\begin{array}{l}\text { Zeiss KL } 2500 \\
\text { LED }\end{array}$ & 635 & 36.8 & 10 & yes & $\geq$ inactivation & [64] \\
\hline $\begin{array}{l}\text { E. dermatitidis, } \\
\text { F. solani, F. } \\
\text { oxysporum } \\
\text { clinical } \\
\text { isolates }\end{array}$ & MB & $\begin{array}{c}\text { itraconazole, } \\
\text { voricona- } \\
\text { zole, } \\
\text { posacona- } \\
\text { zole, } \\
\text { ampho- } \\
\text { tericin }\end{array}$ & $\begin{array}{l}\text { planktonic } \\
\text { and } \\
\text { biofilms }\end{array}$ & LED & $635 \pm 10$ & 100 & $12-24$ & yes & $\begin{array}{c}\text { MIC } \\
\text { decreased }\end{array}$ & [15] \\
\hline $\begin{array}{l}\text { S. globosa } 5 \\
\text { clinical } \\
\text { isolates }\end{array}$ & MB & itraconazole & planktonic & LED & $640 \pm 10$ & 22.2 & 40 & yes & $>$ inactivation & [69] \\
\hline
\end{tabular}

$\left({ }^{*}\right)>$ inactivation: the combination causes an increase in percentage of microbial inactivation greater than the sum of the antibacterial activity of the aPDT plus the antibiotic treatment when they are applied alone. = inactivation: the combination has no effect on the percentage of inactivation; 5-ALA: 5-aminolevulinic acid; aPDT: antimicrobial photodynamic therapy; nd: no data; RB: rose bengal; LED: light-emitting diode; MB: methylene blue; PS: photosensitizer; TMP: meso-tetra ( $N$-methyl-4-pyridyl) porphine tetra tosylate. 
Sporothrix globosa is an etiological agent of sporotrichosis whose most common clinical manifestation is cutaneous and subcutaneous nodular lesions with lymphangitis involvement. Currently, AMR and complications are the major concerns, especially in patients who have liver disorders, children, and pregnant women. Li et al. compared the efficacy in the inactivation of S. globosa of MB-aPDT alone or combined with itraconazole in planktonic culture and in a murine model. The combined treatment offered better results in terms of inactivation percentage and improvement of the lesion size. Therefore, they conclude that MB-aPDT could be an effective adjuvant therapy for resistant infections caused by Sporothrix spp. [69]. In fact, our group treated a patient with cutaneous sporotrichosis using intralesional 1\% MB-aPDT in combination with intermittent low doses of itraconazole obtained complete microbiological and clinical response [70].

\section{Summary of Evidence and Limitations}

In the main, the combination of antibiotics or antifungals with aPDT against in vitro SSTI-etiological agents seems to be beneficial. Combined therapy is more effective than individual treatments alone and often the effects are greater than additivity, i.e., there is synergy. Among the effects reported, the following stand out: (i) the increase in percentage of microbial inactivation; or (ii) the same inactivation percentage is achieved using lower doses of antimicrobials.

It is remarkable that in some cases, drug sensitivity of resistant strains can be restored by combining antibiotics/antifungals with aPDT [15,26,59].

The highlight combinations and the best treatment protocols supported by the existing evidence of in vitro studies on combined aPDT therapies against SSTI-causing agents are included in Table 7. Nevertheless, there is not enough evidence to establish the best combination against each causal agent of SSIT according to this review. The number of studies is limited, and the methodologies used are varied, making direct comparison difficult. In addition, they mostly report the effect on inactivation, but the mechanism of action remains unknown.

This review provides additional and updated information to the one published by Wozniak and Grinholc in 2018, and it is complementary to the review focused on in vivo studies published by our group in 2019 [23,24]. All three types of review agree on the promising approach of combining both therapies and the need to expand knowledge in this line.

The coating of surfaces such as catheters with antimicrobial drugs and aPDT are extremely effective and virtually overcome any resistance build-up. This is more complex on the skin and soft tissues because more variables become part of the process, especially with fungi. However, there is sufficient evidence to support this combined treatment strategy and to lay the foundation for this SSIT treatment approach [18].

Among the obstacles to the incorporation of aPDT as part of the SSIT-treatment, the need to require materials such as lamps for exciting the PS and the need to dedicate more time, because of the irradiation time and because often more than one session of aPDT is required are highlighted. However, it does not require much more clinician specialization, various studies have proven that lamps do not have to be especially expensive and specific, and sources of light with a wide irradiation spectrum or even daylight of radiation can be used effectively $[18,23]$. The methodology and evolution of these treatments need to be reported to the scientific community to continue expanding knowledge and increasingly implement this combination therapeutic option. 
Table 7. The highlight combinations and the best treatment protocols supported by the existing evidence of in vitro studies on combined aPDT therapies against SSTI-causing agents.

$\checkmark \quad$ The combination of different antibiotics with aPDT in general improves the efficacy against in vitro Gram-positive bacteria.

$\checkmark \quad$ Different antibiotics combined with aPDT using porphyrins, phenothiaziniums or RB have synergistic effects in vitro against Gram-positive bacteria being the combination with protoporphyrin the most studied.

$\checkmark \quad$ The combination of aPDT with gentamicin has been extensively tested in both Gram-positive and negative bacteria, reporting positive effects in all cases.

$\checkmark \quad$ The combination of MB-aPDT with sublethal concentrations of antibiotics seems to be a good option against mycobacteria.

$\checkmark \quad$ MB-aPDT combined with ciprofloxacin is the best option in vitro against $E$. coli.

$\checkmark \quad$ Endogenous porphyrins or MB based-aPDT used in combination with antibiotics is a promising option against in vitro $P$. aeruginosa regardless of its antibiotic resistance pattern.

$\checkmark \quad$ The administration of aPDT before antifungals seems to enhance their in vitro antimicrobial effect, especially against yeast and dermatophytes.

aPDT: antimicrobial photodynamic therapy; RB: rose bengal; MB: methylene blue.

\section{Conclusions}

aPTD combined with antimicrobial agents is promising for the management of microorganisms that cause SSTI. It can help to fight them and to overcome AMR.

Author Contributions: Conceptualization and methodology, V.P.-L., Y.G. and A.R.; writing-original draft preparation, V.P.-L.; writing—review and editing V.P.-L., I.G.-L., S.B., Y.G. and A.R.; supervision, Y.G. and A.R.; funding acquisition, I.G.-L., S.B., Y.G. and A.R. All authors have read and agreed to the published version of the manuscript.

Funding: Y.G. And A.R. were founded by Aragon Health Research Institute (IIS Aragón), A.R. was funded by Research Groups of Government of Aragón (Spain) B10_17R Infectious Diseases of Difficult Diagnosis and Treatment. I.G.-L. and S.B. were funded by Plan Nacional de I+D+i and Instituto de Salud Carlos III, Ministerio de Ciencia e Innovación, Spanish Network for Research in Infectious Diseases (REIPI; RD16/0016/0001)-co-financed by European Development Regional Fund "A way to achieve Europe", ERDF.

Institutional Review Board Statement: Not applicable.

Informed Consent Statement: Not applicable.

Data Availability Statement: No new data were created or analyzed in this study. Data sharing is not applicable to this article.

Acknowledgments: The authors thank the research groups of IIS Aragon GIIS-023 and GIS096 for their support.

Conflicts of Interest: The authors declare no conflict of interest. 


\section{References}

1. Ki, V.; Rotstein, C. Bacterial skin and soft tissue infections in adults: A review of their epidemiology, pathogenesis, diagnosis, treatment and site of care. Can. J. Infect. Dis. Med. Microbiol. 2008, 19, 173-184. [CrossRef]

2. Russo, A.; Concia, E.; Cristini, F.; De Rosa, F.G.; Esposito, S.; Menichetti, F.; Petrosillo, N.; Tumbarello, M.; Venditti, M.; Viale, P.; et al. Current and future trends in antibiotic therapy of acute bacterial skin and skin-structure infections. Clin. Microbiol. Infect. 2016, 22 (Suppl. 2), S27-S36. [CrossRef]

3. Sartelli, M.; Guirao, X.; Hardcastle, T.C.; Kluger, Y.; Boermeester, M.A.; Raşa, K.; Ansaloni, L.; Coccolini, F.; Montravers, P.; Abu-Zidan, F.M.; et al. 2018 WSES/SIS-E consensus conference: Recommendations for the management of skin and soft-tissue infections. World J. Emerg. Surg. 2018, 13, 58. [CrossRef] [PubMed]

4. Ibrahim, F.; Khan, T.; Pujalte, G.G.A. Bacterial Skin Infections. Prim. Care 2015, 42, 485-499. [CrossRef] [PubMed]

5. Moffarah, A.S.; Mohajer, M.A.; Hurwitz, B.L.; Armstrong, D.G. Skin and soft tissue infections. Microbiol. Spectr. $2016,4,4$. [CrossRef]

6. Polk, C.; Sampson, M.M.; Roshdy, D.; Davidson, L.E. Skin and Soft Tissue Infections in Patients with Diabetes Mellitus. Infect. Dis. Clin. N. Am. 2021, 35, 183-197. [CrossRef] [PubMed]

7. Esposito, S.; Noviello, S.; de Caro, F.; Boccia, G. New insights into classification, epidemiology and microbiology of SSTIs, including diabetic foot infections. Infez. Med. 2018, 26, 3-14. [PubMed]

8. Leong, H.N.; Kurup, A.; Tan, M.Y.; Kwa, A.L.H.; Liau, K.H.; Wilcox, M.H. Management of complicated skin and soft tissue infections with a special focus on the role of newer antibiotics. Infect. Drug Resist. 2018, 11, 1959-1974. [CrossRef] [PubMed]

9. Stevens, D.L.; Bisno, A.L.; Chambers, H.F.; Dellinger, E.P.; Goldstein, E.J.; Gorbach, S.L.; Hirschmann, J.V.; Kaplan, S.L.; Montoya, J.G.; Wade, J.C.; et al. Practice Guidelines for the Diagnosis and Management of Skin and Soft Tissue Infections: 2014 Update by the Infectious Diseases Society of America. Clin. Infect. Dis. 2014, 59, e10-e52. [CrossRef]

10. Dadgostar, P. Antimicrobial Resistance: Implications and Costs. Infect. Drug Resist. 2019, 12, 3903-3910. [CrossRef]

11. Tacconelli, E. Magrani Global Priority List of Antibiotic-Resistant Bacteria to Guide Research, Discovery, and Development of New Antibiotics; WHO: Geneva, Switzerland, 2017, Available online: https://www.who.int/medicines/publications/WHO-PPLShort_Summary_25Feb-ET_NM_WHO.pdf?ua=1 (accessed on 20 June 2021).

12. Sebeny, P.J.; Riddle, M.S.; Petersen, K. Acinetobacter baumannii skin and soft-tissue infection associated with war trauma. Clin. Infect. Dis. 2008, 47, 444-449. [CrossRef]

13. Berman, J.; Krysan, D.J. Drug resistance and tolerance in fungi. Nat. Rev. Microbiol. 2020, 18, 319-331. [CrossRef] [PubMed]

14. Pappas, P.G.; Rex, J.H.; Sobel, J.D.; Filler, S.G.; Dismukes, W.E.; Walsh, T.J.; Edwards, J.E. Infectious Diseases Society of America Guidelines for treatment of candidiasis. Clin. Infect. Dis. 2004, 38, 161-189. [CrossRef]

15. Gao, L.; Jiang, S.; Sun, Y.; Deng, M.; Wu, Q.; Li, M.; Zeng, T. Evaluation of the Effects of Photodynamic Therapy Alone and Combined with Standard Antifungal Therapy on Planktonic Cells and Biofilms of Fusarium spp. and Exophiala spp. Front. Microbiol. 2016, 7, 617. [CrossRef]

16. Tainwala, R.; Sharma, Y. Pathogenesis of Dermatophytoses. Indian J. Dermatol. 2011, 56, 259-261. [CrossRef] [PubMed]

17. Kharkwal, G.B.; Sharma, S.K.; Huang, Y.-Y.; Dai, T.; Hamblin, M.R. Photodynamic therapy for infections: Clinical applications. Lasers Surg. Med. 2011, 43, 755-767. [CrossRef]

18. Pérez-Laguna, V.; García-Malinis, A.J.; Aspiroz, C.; Rezusta, A.; Gilaberte, Y. Antimicrobial effects of photodynamic therapy: An overview. G. Ital. Dermatol. Venereol. 2018. [CrossRef] [PubMed]

19. Cieplik, F.; Deng, D.; Crielaard, W.; Buchalla, W.; Hellwig, E.; Al-Ahmad, A.; Maisch, T. Antimicrobial photodynamic therapyWhat we know and what we don't. Crit. Rev. Microbiol. 2018, 44, 571-589. [CrossRef] [PubMed]

20. O'Riordan, K.; Akilov, O.E.; Hasan, T. The potential for photodynamic therapy in the treatment of localized infections. Photodiagnosis Photodyn. Ther. 2005, 2, 247-262. [CrossRef]

21. Dai, T.; Huang, Y.-Y.; Hamblin, M.R. Photodynamic therapy for localized infections-state of the art. Photodiagnosis Photodyn. Ther. 2009, 6, 170-188. [CrossRef]

22. Nisnevitch, M.; Valkov, A.; Nakonechny, F.; Gutterman, M.; Nitzan, Y. Antibiotics Combined with Photosensitizers: A Novel Approach to Antibacterial Treatment. In Antibiotic Therapy: New Developments; Turner, A., Hall, J., Eds.; Nova Science Inc.: New York, NY, USA, 2013; pp. 63-88, ISBN 978-1-62808-171-8.

23. Pérez-Laguna, V.; Gilaberte, Y.; Millán-Lou, M.I.; Agut, M.; Nonell, S.; Rezusta, A.; Hamblin, M.R. A combination of photodynamic therapy and antimicrobial compounds to treat skin and mucosal infections: A systematic review. Photochem. Photobiol. Sci. 2019, 18, 1020-1029. [CrossRef] [PubMed]

24. Wozniak, A.; Grinholc, M. Combined Antimicrobial Activity of Photodynamic Inactivation and Antimicrobials-State of the Art. Front. Microbiol. 2018, 9, 930. [CrossRef] [PubMed]

25. Bassetti, M.; Baguneid, M.; Bouza, E.; Dryden, M.; Nathwani, D.; Wilcox, M. European perspective and update on the management of complicated skin and soft tissue infections due to methicillin-resistant Staphylococcus aureus after more than 10 years of experience with linezolid. Clin. Microbiol. Infect. 2014, 20 (Suppl. 4), 3-18. [CrossRef] [PubMed]

26. Ilizirov, Y.; Formanovsky, A.; Mikhura, I.; Paitan, Y.; Nakonechny, F.; Nisnevitch, M. Effect of Photodynamic Antibacterial Chemotherapy Combined with Antibiotics on Gram-Positive and Gram-Negative Bacteria. Molecules 2018, 23, 3152. [CrossRef] [PubMed] 
27. Nieves, I.; Hally, C.; Viappiani, C.; Agut, M.; Nonell, S. A porphycene-gentamicin conjugate for enhanced photodynamic inactivation of bacteria. Bioorganic Chem. 2020, 97, 103661. [CrossRef]

28. Barra, F.; Roscetto, E.; Soriano, A.A.; Vollaro, A.; Postiglione, I.; Pierantoni, G.M.; Palumbo, G.; Catania, M.R. Photodynamic and Antibiotic Therapy in Combination to Fight Biofilms and Resistant Surface Bacterial Infections. Int. J. Mol. Sci. 2015, 16, 20417-20430. [CrossRef] [PubMed]

29. Iluz, N.; Maor, Y.; Keller, N.; Malik, Z. The synergistic effect of PDT and oxacillin on clinical isolates of Staphylococcus aureus. Lasers Surg. Med. 2018, 50, 535-551. [CrossRef]

30. Zhang, Q.-Z.; Zhao, K.-Q.; Wu, Y.; Li, X.-H.; Yang, C.; Guo, L.-M.; Liu, C.-H.; Qu, D.; Zheng, C.-Q. 5-aminolevulinic acid-mediated photodynamic therapy and its strain-dependent combined effect with antibiotics on Staphylococcus aureus biofilm. PLoS ONE 2017, 12, e0174627. [CrossRef]

31. Di Poto, A.; Sbarra, M.S.; Provenza, G.; Visai, L.; Speziale, P. The effect of photodynamic treatment combined with antibiotic action or host defence mechanisms on Staphylococcus aureus biofilms. Biomaterials 2009, 30, 3158-3166. [CrossRef]

32. Dastgheyb, S.S.; Eckmann, D.M.; Composto, R.J.; Hickok, N.J. Photo-activated porphyrin in combination with antibiotics: Therapies against Staphylococci. J. Photochem. Photobiol. B 2013, 129, 27-35. [CrossRef]

33. Branco, T.M.; Valério, N.C.; Jesus, V.I.R.; Dias, C.J.; Neves, M.G.P.M.S.; Faustino, M.A.F.; Almeida, A. Single and combined effects of photodynamic therapy and antibiotics to inactivate Staphylococcus aureus on skin. Photodiagnosis Photodyn. Ther. 2018, 21, 285-293. [CrossRef] [PubMed]

34. Pereira, N.L.F.; Aquino, P.E.A.; Júnior, J.G.A.S.; Cristo, J.S.; Vieira Filho, M.A.; Moura, F.F.; Ferreira, N.M.N.; Silva, M.K.N.; Nascimento, E.M.; Correia, F.M.A.; et al. Antibacterial activity and antibiotic modulating potential of the essential oil obtained from Eugenia jambolana in association with led lights. J. Photochem. Photobiol. B 2017, 174, 144-149. [CrossRef]

35. Ronqui, M.R.; de Aguiar Coletti, T.M.S.F.; de Freitas, L.M.; Miranda, E.T.; Fontana, C.R. Synergistic antimicrobial effect of photodynamic therapy and ciprofloxacin. J. Photochem. Photobiol. B 2016, 158, 122-129. [CrossRef]

36. Kashef, N.; Akbarizare, M.; Razzaghi, M.R. In vitro Activity of Linezolid in Combination with Photodynamic Inactivation Against Staphylococcus aureus Biofilms. Avicenna J. Med. Biotechnol. 2017, 9, 44-48. [PubMed]

37. Liu, S.; Mai, B.; Jia, M.; Lin, D.; Zhang, J.; Liu, Q.; Wang, P. Synergistic antimicrobial effects of photodynamic antimicrobial chemotherapy and gentamicin on Staphylococcus aureus and multidrug-resistant Staphylococcus aureus. Photodiagn. Photodyn. Ther. 2020, 30, 101703. [CrossRef] [PubMed]

38. Pérez-Laguna, V.; García-Luque, I.; Ballesta, S.; Pérez-Artiaga, L.; Lampaya-Pérez, V.; Rezusta, A.; Gilaberte, Y. Photodynamic therapy using methylene blue, combined or not with gentamicin, against Staphylococcus aureus and Pseudomonas aeruginosa. Photodiagn. Photodyn. Ther. 2020, 31, 101810. [CrossRef] [PubMed]

39. Pérez-Laguna, V.; Pérez-Artiaga, L.; Lampaya-Pérez, V.; García-Luque, I.; Ballesta, S.; Nonell, S.; Paz-Cristobal, M.P.; Gilaberte, Y.; Rezusta, A. Bactericidal Effect of Photodynamic Therapy, Alone or in Combination with Mupirocin or Linezolid, on Staphylococcus aureus. Front. Microbiol. 2017, 8, 1002. [CrossRef]

40. Pérez, M.; Robres, P.; Moreno, B.; Bolea, R.; Verde, M.T.; Pérez-Laguna, V.; Aspiroz, C.; Gilaberte, Y.; Rezusta, A. Comparison of Antibacterial Activity and Wound Healing in a Superficial Abrasion Mouse Model of Staphylococcus aureus Skin Infection Using Photodynamic Therapy Based on Methylene Blue or Mupirocin or Both. Front. Med. 2021, 8. [CrossRef]

41. Pérez-Laguna, V.; García-Luque, I.; Ballesta, S.; Pérez-Artiaga, L.; Lampaya-Pérez, V.; Samper, S.; Soria-Lozano, P.; Rezusta, A.; Gilaberte, Y. Antimicrobial photodynamic activity of Rose Bengal, alone or in combination with Gentamicin, against planktonic and biofilm Staphylococcus aureus. Photodiagn. Photodyn. Ther. 2018, 21, 211-216. [CrossRef] [PubMed]

42. de Allori, M.C.G.; Jure, M.A.; Romero, C.; de Castillo, M.E.C. Antimicrobial resistance and production of biofilms in clinical isolates of coagulase-negative Staphylococcus strains. Biol. Pharm. Bull. 2006, 29, 1592-1596. [CrossRef]

43. Fey, P.D.; Olson, M.E. Current concepts in biofilm formation of Staphylococcus epidermidis. Future Microbiol. 2010, 5, 917-933. [CrossRef]

44. Foster, T. Staphylococcus. In Medical Microbiology, 4th ed.; Baron, S., Ed.; University of Texas Medical Branch at Galveston: Galveston, TX, USA, 1996. Available online: http:/ /www.ncbi.nlm.nih.gov/books/NBK8448/ (accessed on 10 July 2018).

45. Viale, P.; Stefani, S. Vascular catheter-associated infections: A microbiological and therapeutic update. J. Chemother. Florence Italy 2006, 18, 235-249. [CrossRef]

46. Sbarra, M.S.; Arciola, C.R.; Di Poto, A.; Saino, E.; Rohde, H.; Speziale, P.; Visai, L. The photodynamic effect of tetra-substituted N-methyl-pyridyl-porphine combined with the action of vancomycin or host defense mechanisms disrupts Staphylococcus epidermidis biofilms. Int. J. Artif. Organs 2009, 32, 574-583. [CrossRef] [PubMed]

47. Gong, N.; Tan, Y.; Li, M.; Lu, W.; Lei, X. ALA-PDT combined with antibiotics for the treatment of multiple skin abscesses caused by Mycobacterium fortuitum. Photodiagn. Photodyn. Ther. 2016, 15, 70-72. [CrossRef] [PubMed]

48. Sun, K.; Yang, H.; Huang, X.; Gong, N.; Qin, Q.; Lu, W.; Lei, X. ALA-PDT combined with antibiotics for the treatment of atypical mycobacterial skin infections: Outcomes and safety. Photodiagnosis Photodyn. Ther. 2017, 19, 274-277. [CrossRef] [PubMed]

49. Shih, M.-H.; Huang, F.-C. Effects of photodynamic therapy on rapidly growing nontuberculous mycobacteria keratitis. Investig. Ophthalmol. Vis. Sci. 2011, 52, 223-229. [CrossRef]

50. Boucher, H.W.; Talbot, G.H.; Bradley, J.S.; Edwards, J.E.; Gilbert, D.; Rice, L.B.; Scheld, M.; Spellberg, B.; Bartlett, J. Bad bugs, no drugs: No ESKAPE! An update from the Infectious Diseases Society of America. Clin. Infect. Dis. 2009, 48, 1-12. [CrossRef] [PubMed] 
51. Tichaczek-Goska, D.; Wojnicz, D.; Symonowicz, K.; Ziółkowski, P.; Hendrich, A.B. Photodynamic enhancement of the activity of antibiotics used in urinary tract infections. Lasers Med. Sci. 2019. [CrossRef] [PubMed]

52. Almeida, J.; Tomé, J.P.C.; Neves, M.G.P.M.S.; Tomé, A.C.; Cavaleiro, J.A.S.; Cunha, Â.; Costa, L.; Faustino, M.A.F.; Almeida, A. Photodynamic inactivation of multidrug-resistant bacteria in hospital wastewaters: Influence of residual antibiotics. Photochem. Photobiol. Sci. 2014, 13, 626-633. [CrossRef]

53. Magacho, C.C.; Pinto, J.G.; Souza, B.M.N.; Pereira, A.H.C.; Ferreira-Strixino, J. Comparison of photodynamic therapy with methylene blue associated with ceftriaxone in gram-negative bacteria; an in vitro study. Photodiagn. Photodyn. Ther. 2020, 30, 101691. [CrossRef]

54. Collins, T.L.; Markus, E.A.; Hassett, D.J.; Robinson, J.B. The effect of a cationic porphyrin on Pseudomonas aeruginosa biofilms. Curr. Microbiol. 2010, 61, 411-416. [CrossRef] [PubMed]

55. Reznick, Y.; Banin, E.; Lipovsky, A.; Lubart, R.; Polak, P.; Zalevsky, Z. The synergistic effect of visible light and gentamycin on Pseudomona aeruginosa microorganisms. J. Vis. Exp. JoVE 2013, e4370. [CrossRef] [PubMed]

56. Fila, G.; Kawiak, A.; Grinholc, M.S. Blue light treatment of Pseudomonas aeruginosa: Strong bactericidal activity, synergism with antibiotics and inactivation of virulence factors. Virulence 2017, 8, 938-958. [CrossRef] [PubMed]

57. Oppezzo, O.J.; Forte Giacobone, A.F. Lethal Effect of Photodynamic Treatment on Persister Bacteria. Photochem. Photobiol. 2018, 94, 186-189. [CrossRef] [PubMed]

58. Nakonieczna, J.; Wolnikowska, K.; Ogonowska, P.; Neubauer, D.; Bernat, A.; Kamysz, W. Rose Bengal-Mediated Photoinactivation of Multidrug Resistant Pseudomonas aeruginosa Is Enhanced in the Presence of Antimicrobial Peptides. Front. Microbiol. 2018, 9. [CrossRef] [PubMed]

59. Wozniak, A.; Rapacka-Zdonczyk, A.; Mutters, N.T.; Grinholc, M. Antimicrobials Are a Photodynamic Inactivation Adjuvant for the Eradication of Extensively Drug-Resistant Acinetobacter baumannii. Front. Microbiol. 2019, 10, 229. [CrossRef]

60. Kashem, S.W.; Kaplan, D.H. Skin Immunity to Candida albicans. Trends Immunol. 2016, 37, 440-450. [CrossRef]

61. Lyon, J.P.; Carvalho, C.R.; Rezende, R.R.; Lima, C.J.; Santos, F.V.; Moreira, L.M. Synergism between fluconazole and methylene blue-photodynamic therapy against fluconazole-resistant Candida strains. Indian J. Med. Microbiol. 2016, 34, 506-508. [CrossRef]

62. Snell, S.B.; Foster, T.H.; Haidaris, C.G. Miconazole induces fungistasis and increases killing of Candida albicans subjected to photodynamic therapy. Photochem. Photobiol. 2012, 88, 596-603. [CrossRef]

63. Morton, C.O.; Chau, M.; Stack, C. In vitro combination therapy using low dose clotrimazole and photodynamic therapy leads to enhanced killing of the dermatophyte Trichophyton rubrum. BMC Microbiol. 2014, 14, 261. [CrossRef]

64. Hu, Y.; Qi, X.; Sun, H.; Lu, Y.; Hu, Y.; Chen, X.; Liu, K.; Yang, Y.; Mao, Z.; Wu, Z.; et al. Photodynamic therapy combined with antifungal drugs against chromoblastomycosis and the effect of ALA-PDT on Fonsecaea in vitro. PLoS Negl. Trop. Dis. 2019, 13, e0007849. [CrossRef] [PubMed]

65. Hu, Y.; Huang, X.; Lu, S.; Hamblin, M.R.; Mylonakis, E.; Zhang, J.; Xi, L. Photodynamic therapy combined with terbinafine against chromoblastomycosis and the effect of PDT on Fonsecaea monophora in vitro. Mycopathologia 2015, 179, 103-109. [CrossRef] [PubMed]

66. Yang, Y.; Hu, Y.; Zhang, J.; Li, X.; Lu, C.; Liang, Y.; Xi, L. A refractory case of chromoblastomycosis due to Fonsecaea monophora with improvement by photodynamic therapy. Med. Mycol. 2012, 50, 649-653. [CrossRef] [PubMed]

67. Guarro, J. Fusariosis, a complex infection caused by a high diversity of fungal species refractory to treatment. Eur. J. Clin. Microbiol. Infect. Dis. 2013, 32, 1491-1500. [CrossRef]

68. Giroldo, L.M.; Felipe, M.P.; de Oliveira, M.A.; Munin, E.; Alves, L.P.; Costa, M.S. Photodynamic antimicrobial chemotherapy (PACT) with methylene blue increases membrane permeability in Candida albicans. Lasers Med. Sci. 2009, 24, 109-112. [CrossRef]

69. Li, J.; Zhu, M.; An, L.; Chen, F.; Zhang, X. Fungicidal efficacy of photodynamic therapy using methylene blue against Sporothrix globosa in vitro and in vivo. Eur. J. Dermatol. EJD 2019, 29, 160-166. [CrossRef]

70. Gilaberte, Y.; Aspiroz, C.; Alejandre, M.C.; Andres-Ciriano, E.; Fortuño, B.; Charlez, L.; Revillo, M.J.; Hamblin, M.R.; Rezusta, A. Cutaneous Sporotrichosis Treated with Photodynamic Therapy: An in Vitro and in Vivo Study. Photomed. Laser Surg. 2014, 32, 54-57. [CrossRef] 